\title{
Semigroup Maximal Functions, Riesz Transforms, and Morrey Spaces Associated with Schrödinger Operators on the Heisenberg Groups
}

\author{
Hua Wang \\ School of Mathematics and Systems Science, Xinjiang University, Urumqi 830046, China \\ Correspondence should be addressed to Hua Wang; wanghua@pku.edu.cn
}

Received 8 August 2020; Accepted 28 October 2020; Published 21 November 2020

Academic Editor: Eva A. Gallardo Gutiérrez

Copyright (C) 2020 Hua Wang. This is an open access article distributed under the Creative Commons Attribution License, which permits unrestricted use, distribution, and reproduction in any medium, provided the original work is properly cited.

\begin{abstract}
Let $\mathscr{L}=-\Delta_{\mathbb{H}^{n}}+V$ be a Schrödinger operator on the Heisenberg group $\mathbb{H}^{n}$, where $\Delta_{\mathbb{H}^{n}}$ is the sub-Laplacian on $\mathbb{H}^{n}$ and the nonnegative potential $V$ belongs to the reverse Hölder class $\mathscr{B}_{q}$ with $q \in[Q / 2, \infty)$. Here, $Q=2 n+2$ is the homogeneous dimension of $\mathbb{H}^{n}$. Assume that $\left\{e^{-t \mathscr{L}}\right\}_{t>0}$ is the heat semigroup generated by $\mathscr{L}$. The semigroup maximal function related to the Schrödinger operator $\mathscr{L}$ is defined by $\mathscr{T}_{\mathscr{L}}^{*}(f)(u):=\sup _{t>0}\left|e^{-t \mathscr{L}} f(u)\right|$. The Riesz transform associated with the operator $\mathscr{L}$ is defined by $\mathscr{R}_{\mathscr{L}}=\nabla_{\mathbb{H}^{n}} \mathscr{L}^{-1 / 2}$, and the dual Riesz transform is defined by $\mathscr{R}_{\mathscr{L}}^{*}=\mathscr{L}^{-1 / 2} \nabla_{\mathbb{H}^{n}}$, where $\nabla_{\mathbb{H}^{n}}$ is the gradient operator on $\mathbb{H}^{n}$. In this paper, the author first introduces a class of Morrey spaces associated with the Schrödinger operator $\mathscr{L}$ on $\mathbb{H}^{n}$. Then, by using some pointwise estimates of the kernels related to the nonnegative potential, the author establishes the boundedness properties of these operators $\mathscr{T}_{\mathscr{L}}^{*}, \mathscr{R}_{\mathscr{L}}$, and $\mathscr{R}_{\mathscr{L}}^{*}$ acting on the Morrey spaces. In addition, it is shown that the Riesz transform $\mathscr{R}_{\mathscr{L}}=\nabla_{\mathbb{H}^{n}} \mathscr{L}^{-1 / 2}$ is of weak-type $(1,1)$. It can be shown that the same conclusions are also true for these operators on generalized Morrey spaces.
\end{abstract}

\section{Introduction}

1.1. The Heisenberg Group $\mathbb{H}^{n}$. The Heisenberg group is the most well-known example from the realm of nilpotent Lie groups and plays an important role in several branches of mathematics, such as the representation theory, partial differential equations, several complex variables, and harmonic analysis. It is a remarkable fact that the Heisenberg group, an important example of the simply connected nilpotent Lie group, naturally arises in two fundamental but different settings in modern analysis. On the one hand, it can be naturally identified with the group of translations of the Siegel upper half-space in $\mathbb{C}^{n+1}$ and plays an important role in our understanding of several problems in the complex function theory of the unit ball. On the other hand, it can also be realized as the group of unitary operators generated by the position and momentum operators in the context of quantum mechanics.
We begin by recalling some notions and well-known results from [1-3]. We write $\mathbb{N}=\{1,2,3, \cdots\}$ for the set of natural numbers. The sets of real and complex numbers are denoted by $\mathbb{R}$ and $\mathbb{C}$, respectively. Let $\mathbb{H}^{n}$ be a Heisenberg group of dimension $2 n+1$, that is, a two-step nilpotent Lie group with underlying manifold $\mathbb{C}^{n} \times \mathbb{R}$. The group operation is given by

$$
(z, t) \bullet(w, s):=(z+w, t+s+2 \operatorname{Im}(z \bullet \bar{w}))
$$

where $z=\left(z_{1}, z_{2}, \cdots, z_{n}\right), w=\left(w_{1}, w_{2}, \cdots, w_{n}\right) \in \mathbb{C}^{n}$, and $z \bullet$ $\bar{w}:=\sum_{j=1}^{n} z_{j} \overline{w_{j}}$.

Under this group operation, $\mathbb{H}^{n}$ becomes a nilpotent unimodular Lie group. It can easily be seen that the inverse element of $u=(z, t) \in \mathbb{H}^{n}$ is $u^{-1}=(-z,-t)$, and the identity element of this group is the origin $0=(0,0)$. The corresponding Lie algebra $\mathfrak{H}^{n}$ of left-invariant vector fields on $\mathbb{H}^{n}$ is 
spanned by

$$
X_{j}:=\frac{\partial}{\partial x_{j}}+2 y_{j} \frac{\partial}{\partial t} ; Y_{j}:=\frac{\partial}{\partial y_{j}}-2 x_{j} \frac{\partial}{\partial t} ; \quad j=1,2, \cdots, n, T:=\frac{\partial}{\partial t} .
$$

All nontrivial commutation relations are given by

$$
\left[X_{j}, Y_{j}\right]=-4 T, \quad j=1,2, \cdots, n
$$

Here, $[\cdot, \cdot]$ is the usual Lie bracket. The sub-Laplacian $\Delta_{\mathbb{H}^{n}}$ and the gradient $\nabla_{\mathbb{H}^{n}}$ are defined, respectively, by

$$
\begin{aligned}
\Delta_{\mathbb{H}^{n}} & :=\sum_{j=1}^{n}\left(X_{j}^{2}+Y_{j}^{2}\right), \\
\nabla_{\mathbb{H}^{n}} & :=\left(X_{1}, \cdots, X_{n}, Y_{1}, \cdots, Y_{n}\right) .
\end{aligned}
$$

The Heisenberg group has a natural dilation structure which is consistent with the Lie group structure mentioned above. For each positive number $a>0$, we define the dilation on $\mathrm{H}^{n}$ by

$$
\delta_{a}(z, t):=\left(a z, a^{2} t\right), \quad(z, t) \in \mathbb{H}^{n}
$$

Observe that $\delta_{a}(a>0)$ is an automorphism of the group $\mathbb{H}^{n}$. For any given $u=(z, t) \in \mathbb{H}^{n}$, the homogeneous norm of $u$ is given by the following form:

$$
|u|=|(z, t)|:=\left(|z|^{4}+t^{2}\right)^{1 / 4} \text {. }
$$

Observe that $\left|(z, t)^{-1}\right|=|(z, t)|$ and

$$
\left|\delta_{a}(z, t)\right|=\left(|a z|^{4}+\left(a^{2} t\right)^{2}\right)^{1 / 4}=a|(z, t)|, \quad a>0
$$

In addition, this norm $|\cdot|$ satisfies the triangle inequality and then leads to a left-invariant distance $d(u, v)=\left|u^{-1} \cdot v\right|$ for any $u=(z, t), v=(w, s) \in \mathbb{H}^{n}$. If $r>0$ and $u \in \mathbb{H}^{n}$, let $B(u, r)=\left\{v \in \mathbb{H}^{n}: d(u, v)<r\right\}$ be the (open) ball with center $u \in \mathbb{H}^{n}$ and radius $r \in(0, \infty)$. Both left and right Haar measures on $\mathbb{H}^{n}$ coincide with the Lebesgue measure $d z d t$ on $\mathbb{C}^{n} \times \mathbb{R}$. For any measurable set $E \subset \mathbb{H}^{n}$, the Lebesgue measure of $E$ is denoted by $|E|$. For $(u, r) \in \mathbb{H}^{n} \times(0, \infty)$, it can be proved that the volume of $B(u, r)$ is

$$
|B(u, r)|=r^{Q}|B(0,1)|
$$

where $Q:=2_{n}+2$ is the homogeneous dimension of $\mathbb{H}^{n}$ and $|B(0,1)|$ is the volume of the unit ball in $\mathbb{H}^{n}$. A simple calculation shows that

$$
|B(0,1)|=\frac{2 \pi^{n+(1 / 2) \Gamma(n / 2)}}{(n+1) \Gamma((n+1) / 2)} .
$$

Given a ball $B=B(u, r) \subset \mathbb{H}^{n}$ and $\lambda>0$, we adopt the notation $\lambda B$ to denote the ball with the same center $u$ and radius $\lambda r$. Clearly, by (8), we have

$$
|B(u, \lambda r)|=\lambda^{Q}|B(u, r)|,(u, r) \in \mathbb{H}^{n} \times(0, \infty), \lambda \in(0, \infty) .
$$

For a radial function $F$ on $\mathbb{H}^{n}$, we have the following integration formula:

$$
\int_{\mathbb{H}^{n}} F(u) d u=C_{n} \int_{0}^{\infty} F(\varrho) \varrho^{Q-1} d \varrho, Q=2 n+2,
$$

where $C_{n}$ is a positive constant which is independent of $F$. For more information about harmonic analysis on the Heisenberg group, the reader is referred to $[2,4,5]$ and the references therein.

1.2. The Schrödinger Operator $\mathscr{L}$. We recall some standard notation and definitions.

Definition 1. A nonnegative locally $L^{q}$ integrable function $V$ on $\mathrm{H}^{n}$ is said to belong to the reverse Hölder class $\mathscr{B}_{q}$ for some exponent $1<q<\infty$, if there exists a positive constant $C=C(q ; V)$ such that the reverse Hölder inequality

$$
\left(\frac{1}{|B|} \int_{B} V(w)^{q} d w\right)^{1 / q} \leq C\left(\frac{1}{|B|} \int_{B}(w) d w\right)
$$

holds for every ball $B$ in $\mathbb{H}^{n}$.

In this article, we will always assume that $0 \equiv V \in B_{q}$ with $q \in[Q / 2, \infty)$ and $Q=2 n+2$. We now consider the Schrödinger operator with the potential $V \in \mathscr{B}_{q}$ on the Heisenberg group $\mathbb{H}^{n}$ (see [3]):

$$
\mathscr{L}:=-\Delta_{\mathbb{H}} n+V
$$

In recent years, there has been a lot of attention paid to the study of various function spaces associated with the Schrödinger operators, which has been an active research topic in harmonic analysis. For the investigation of Schrödinger operators on the Euclidean space $\mathbb{R}^{n}$ with nonnegative potentials that belong to the reverse Hölder class, see, for example, [6-10]. Concerning the weighted case, one can see [11-16] for more details. The extension to the setting of the Heisenberg group has been given by Lin and Liu in [3]. For further details, we refer the reader to [17-19], among others. Regarding the Schrödinger operators in a more general setting (such as the nilpotent Lie group), see, for example, $[20,21]$. As in $[3,22]$, we introduce the following definition.

Definition 2. Suppose that $V \in \mathscr{B}_{q}$ with $q \in[Q / 2, \infty)$. For any given $u \in \mathbb{H}^{n}$, the critical radius function $\rho(u)=\rho(u ; V)$ is defined by

$$
\rho(u):=\left\{r \in(0, \infty): \frac{1}{r^{Q-2}} \int_{B(u, r)} V(w) d w \leq 1\right\},
$$


where $B(u, r)$ denotes the ball in $\mathbb{H}^{n}$ centered at $u$ and with radius $r$.

It should be pointed out that the auxiliary function $\rho$ $(u ; V)$ on the Euclidean space $\mathbb{R}^{n}$ was introduced by Shen in [10]. Later, Li [21] defined it on the (simply connected) nilpotent Lie group. It is well known that the auxiliary function $\rho(u)$ determined by $V \in \mathscr{B}_{q}$ satisfies

$$
0<\rho(u)<\infty
$$

for any given $u \in \mathbb{H}^{n}$ (see $[3,22]$ ). In particular, $\rho(u)=1$ with $V(w) \equiv 1$, and $\rho(u) \approx 1 /(1+|u|)$ with $V(w)=|w|^{2}$ (Hermite operator).

It is easy to check that if $Q / 2 \leq q_{2}<q_{1}$, then $\mathscr{B}_{q_{1}} \subset \mathscr{B}_{q_{2}}$ by the Hölder inequality. Furthermore, it can be shown that the $\mathscr{B}_{q}$ class has a property of self-improvement. More precisely, if $V \in \mathscr{B}_{q}$, then $V \in \mathscr{B}_{q+\varepsilon}$ for some $\varepsilon>0$. By this fact, we know that the assumption $q>Q / 2$ is equivalent to $q \geq Q / 2$.

When $q \in[Q / 2, Q)$, we also write

$$
\delta:=2-\frac{Q}{q} \in(0,1)
$$

Let us give some elementary properties of the $\mathscr{B}_{q}$ class. Assume that $V \in \mathscr{B}_{q}$ with $q \in[Q / 2, \infty)$.

Lemma 3. The measure $V(w) d w$ satisfies the doubling condition; that is, there exists a constant $C_{0}>0$ such that

$$
\int_{B\left(u_{0}, 2 r\right)} V(w) d w \leq C_{0} \int_{B\left(u_{0}, r\right)} V(w) d w
$$

for all balls $B\left(u_{0}, r\right)$ in $\mathbb{H}^{n}$.

Lemma 4. If $r=\rho(u 0)$, then

$$
\frac{1}{r^{Q-2}} \int_{B\left(u_{0}, r\right)} V(w) d w=1,
$$

for any $u_{0} \in \mathbb{H}^{n}$.

Lemma 5. For $0<r<R<\infty$, we have

$$
\frac{1}{r^{Q-2}} \int_{B\left(u_{0}, r\right)} V(w) d w \leq C\left(\frac{r}{R}\right)^{\delta} \frac{1}{R^{Q-2}} \int_{B\left(u_{0}, R\right)} V(w) d w,
$$

for any $u_{0} \in \mathbb{H}^{n}$.

For more details, the reader may consult $[3,22]$.

We also need the following technical lemma concerning the critical radius function (14).

Lemma 6. Let $\rho(u)$ be the auxiliary function determined by $V$. For any $u$ and $v$ in $\mathbb{H}^{n}$, there exist constants $C_{1} \geq 1$ and $N_{0}>0$ such that

$$
\frac{1}{C_{1}}\left[1+\frac{\left|v^{-1} u\right|}{\rho(u)}\right]^{-N_{0}} \leq \frac{\rho(v)}{\rho(u)} \leq C_{1}\left[1+\frac{\left|v^{-1} u\right|}{\rho(u)}\right]^{N_{0} /\left(N_{0}+1\right)} .
$$

Here, and in what follows, $v^{-1} \cdot u$ is simply denoted by $v^{-1} u$. Lemma 6 has been proved by Lu [22] (see also [3], Lemma 4). In the setting of $\mathbb{R}^{n}$, this result was first given by Shen in [10] (Lemma 1.4). As a direct consequence of (20), we can see that for each fixed $k \in \mathbb{N}$, the following estimate

$$
\left[1+\frac{2^{k} r}{\rho(u)}\right]\left[1+\frac{r}{\rho(u)}\right]^{-N_{0} /\left(N_{0}+1\right)} \leq C_{1}\left[1+\frac{2^{k} r}{\rho(v)}\right],
$$

holds for any $v \in B(u, r)$ with $u \in \mathbb{H}^{n}$ and $r \in(0, \infty)$, where $C_{1}$ is the same as in (20). Let us verify (21). An application of (20) yields

$$
\begin{aligned}
\rho(v) & \leq C_{1} \cdot \rho(u)\left[1+\frac{\left|v^{-1} u\right|}{\rho(u)}\right]^{N_{0} /\left(N_{0}+1\right)} \\
& \leq C_{1} \cdot \rho(u)\left[1+\frac{r}{\rho(u)}\right]^{N_{0} /\left(N_{0}+1\right)}
\end{aligned}
$$

which further implies that for each fixed $k \in \mathbb{N}$,

$$
\frac{2^{k} r}{\rho(v)} \geq \frac{1}{C_{1}} \cdot \frac{2^{k} r}{\rho(u)}\left[1+\frac{r}{\rho(u)}\right]^{-N_{0} /\left(N_{0}+1\right)} .
$$

Hence,

$$
\begin{aligned}
{\left[1+\frac{2^{k} r}{\rho(v)}\right] \geq } & 1+\frac{1}{C_{1}} \cdot \frac{2^{k} r}{\rho(u)}\left[1+\frac{r}{\rho(u)}\right]^{-N_{0} /\left(N_{0}+1\right)} \\
\geq & \frac{1}{C_{1}}\left[1+\frac{r}{\rho(u)}\right]^{-N_{0} /\left(N_{0}+1\right)}+\frac{1}{C_{1}} \\
& \cdot \frac{2^{k} r}{\rho(u)}\left[1+\frac{r}{\rho(u)}\right]^{-N_{0} /\left(N_{0}+1\right)} \\
= & \frac{1}{C_{1}}\left[1+\frac{2^{k} r}{\rho(u)}\right]\left[1+\frac{r}{\rho(u)}\right]^{-N_{0} /\left(N_{0}+1\right)},
\end{aligned}
$$

which is the desired estimate. This estimate will often be used in the sequel.

1.3. Semigroup Maximal Functions and Riesz Transforms. Let $\mathscr{L}=-\Delta_{\mathbb{H}^{n}}+V$ be a Schrödinger operator on the Heisenberg group $\mathbb{H}^{n}$, where $\Delta_{\mathbb{H}^{n}}$ is the sub-Laplacian and the nonnegative potential $V$ belongs to the reverse Hölder class $\mathscr{B}_{q}$ for $q \in[Q / 2, \infty)$, and $Q$ is the homogeneous dimension of $\mathrm{H}^{n}$. Since $V$ is nonnegative and belongs to $L_{\text {loc }}^{q}\left(\mathbb{H}^{n}\right), \mathscr{L}$ generates a $\left(\mathscr{C}_{0}\right)$ contraction semigroup $\left\{\mathscr{T}_{t}^{\mathscr{L}}\right\}_{t>0}=\left\{e^{-t \mathscr{L}}\right\}_{t>0}$. Let $\mathscr{P}_{t}$ 
$(u, v)$ denote the kernel of the semigroup $\left\{e^{-t \mathscr{L}}\right\}_{t>0}$.

$\mathscr{T}_{t}^{\mathscr{L}} f(u):=e^{-t \mathscr{L}} f(u)=\int_{\mathbb{H}^{n}} \mathscr{P}_{t}(u, v) f(v) d v, \quad f \in L^{2}\left(\mathbb{H}^{n}\right), t>0$.

We also denote by $H_{t}(u)$ the convolution kernel of the heat semigroup $\left\{T_{t}\right\}_{t>0}=\left\{e^{t \Delta_{\mathbb{H}^{n}}}\right\}_{t>0}$. Namely,

$$
T_{t} f(u):=e^{t \Delta_{\mathbb{H}^{n}}} f(u)=\int_{\mathbb{H}^{n}} H_{t}\left(v^{-1} u\right) f(v) d v
$$

For any $u=(z, s) \in \mathbb{H}^{n}$, it is well known that the heat kernel $H_{t}(u)$ has the explicit expression:

$$
\begin{aligned}
H_{t}(z, s)= & (2 \pi)^{-1}(4 \pi)^{-n} \int_{\mathbb{R}} \\
& \cdot\left(\frac{|\lambda|}{\sinh |\lambda| t}\right)^{n} \exp \left\{-\frac{|\lambda||z|^{2}}{4} \operatorname{coth}|\lambda| t-i \lambda s\right\} d \lambda .
\end{aligned}
$$

We consider the heat equation associated with the subLaplacian

$$
\partial_{t} F(u, t)=\Delta_{\mathbb{H}^{n}} F(u, t), \quad(u, t) \in \mathbb{H}^{n} \times(0, \infty),
$$

with the initial condition $F(u, 0)=f(u)$. In fact, the function $H_{t}(u)$ stated the above exists as a solution to the heat equation. Moreover, by [23] (Theorem 2), we know that the heat kernel $H_{t}$ satisfies the Gaussian upper bound estimate:

$$
0<H_{t}(u) \leq C \cdot t^{-Q / 2} \exp \left(-\frac{|u|^{2}}{A t}\right)
$$

where the positive constants $C$ and $A$ are independent of $t$ $\in(0, \infty)$ and $u \in \mathbb{H}^{n}$. By the Trotter product formula (see [24] for instance) and (29), one has

$$
0 \leq \mathscr{P}_{t}(u, v) \leq H_{t}\left(v^{-1} u\right) \leq C \cdot t^{-Q / 2} \exp \left(-\frac{\left|v^{-1} u\right|^{2}}{A t}\right)
$$

where $C$ and $A$ are positive constants independent of $u, v$, and $t$. Furthermore, by using the estimates of the fundamental solution for the Schrödinger operator $\mathscr{L}$ on $\mathbb{H}^{n}$, this estimate (30) can be significantly improved when $V$ belongs to the reverse Hölder class $\mathscr{B}_{q}$ for some $q \in[Q / 2, \infty)$. The auxiliary function $\rho(u)$ arises naturally in the present situation.

Lemma 7. Let $\rho(u)$ be the auxiliary function determined by $V$. For every positive integer $N \in \mathbb{N}$, there exists a positive con- stant $C_{N}>0$ such that, for any $u$ and $v$ in $\mathrm{H}^{n}$,

$$
\begin{aligned}
0 & \leq \mathscr{P}_{t}(u, v) \\
& \leq C_{N} \cdot t^{-Q / 2} \exp \left(-\frac{\left|v^{-1} u\right|^{2}}{A t}\right)\left[1+\frac{\sqrt{t}}{\rho(u)}+\frac{\sqrt{t}}{\rho(v)}\right]^{-N}, \quad t>0 .
\end{aligned}
$$

Remark 8. This estimate of $\mathscr{P}_{t}(u, v)$ is much better than (30), which was given by Lin and Liu in [3] (Lemma 7). In the setting of $\mathbb{R}^{n}$, this result can be found in [9] (Proposition 2).

In this article, we investigate the semigroup maximal function related to the Schrödinger operator $\mathscr{L}$, which is defined by (see [3])

$$
\mathscr{T}_{\mathscr{L}}^{*}(f)(u):=\sup _{t>0}\left|\mathscr{T}_{t}^{\mathscr{L}} f(u)\right|=\sup _{t>0}\left|e^{-t \mathscr{L}} f(u)\right|, u \in \mathbb{H}^{n}
$$

We shall establish the strong-type and weak-type estimates of the operator $\mathscr{T}_{\mathscr{L}}^{*}$. Some other maximal functions will be discussed at the end of Section 3.

Let us also consider the Riesz transforms $\mathscr{R}_{j}$ and the dual Riesz transforms $\mathscr{R}_{j}^{*}$ for the Schrödinger operator $\mathscr{L}$, which are defined, respectively, by (see [3])

$$
\left\{\begin{array}{l}
\mathscr{R}_{j}=X_{j} \mathscr{L}^{-1 / 2}, \mathscr{R}_{j+1}=Y_{j} \mathscr{L}^{-1 / 2}, j=1,2, \cdots, n, \\
\mathscr{R}_{j}^{*}=\mathscr{L}^{-1 / 2} X_{j}, \mathscr{R}_{j+1}^{*}=\mathscr{L}^{-1 / 2} Y_{j}, j=1,2, \cdots, n,
\end{array}\right.
$$

where the $X_{j}$ are left-invariant vector fields that generate the Lie algebra of $\mathbb{H}^{n}$. Let

$$
\begin{aligned}
& \mathscr{R}_{\mathscr{L}}:=\nabla_{\mathbb{H}^{n}} \mathscr{L}^{-1 / 2}=\left(\mathscr{R}_{1}, \mathscr{R}_{2}, \cdots, \mathscr{R}_{2 n}\right), \\
& \mathscr{R}_{\mathscr{L}}^{*}:=\mathscr{L}^{-1 / 2} \nabla_{\mathbb{H}^{n}}=\left(\mathscr{R}_{1}^{*}, \mathscr{R}_{2}^{*}, \cdots, \mathscr{R}_{2 n}^{*}\right) .
\end{aligned}
$$

Here, $\nabla_{\mathbb{H}^{n}}$ is the gradient operator on $\mathbb{H}^{n}$. We shall be interested in the behavior of the (vector-valued) operators $\mathscr{R}_{\mathscr{L}}$ and $\mathscr{R}_{\mathscr{L}}^{*}$ associated with the Schrödinger operator $\mathscr{L}$ on $\mathbb{H}^{n}$.

For any $p \in[1, \infty)$, the Lebesgue space $L^{p}\left(\mathbb{H}^{n}\right)$ is defined to be the set of all measurable functions $f$ on $\mathbb{H}^{n}$ such that

$$
\|f\|_{L^{p}\left(\mathbb{H}^{n}\right)}:=\left(\int_{\mathbb{H}^{n}}|f(u)|^{p} d u\right)^{1 / p}<\infty
$$

The weak Lebesgue space $W L^{1}\left(\mathbb{H}^{n}\right)$ consists of all measurable functions $f$ on $\mathbb{H}^{n}$ such that

$$
\|f\|_{W L^{1}\left(\mathbb{H}^{n}\right)}:=\sup _{\lambda>0} \lambda \cdot\left|\left\{u \in \mathbb{H}^{n}:|f(u)|>\lambda\right\}\right|<\infty .
$$

Recently, Lin and Liu ([3], Theorem 6 and Remark 3) established the strong-type and weak-type estimates of the operator $\mathscr{T}_{\mathscr{L}}^{*}$ on the Lebesgue spaces. 
Theorem 9. Let $1 \leq p<\infty$. Then, the following statements are true:

(1) If $p>1$, then the operator $\mathscr{T}_{\mathscr{L}}^{*}$ is bounded on $L^{p}\left(\mathbb{H}^{n}\right)$

(2) If $p=1$, then the operator $\mathscr{T}_{\mathscr{L}}^{*}$ is bounded from $L^{1}\left(\mathbb{H}^{n}\right)$ into $W L^{1}\left(\mathbb{H}^{n}\right)$

Remark 10.

(i) It was also shown by Lin and Liu that this operator is bounded on $\mathrm{BMO}_{\mathscr{L}}\left(\mathbb{H}^{n}\right)$

(ii) On the Euclidean space $\mathbb{R}^{n}$, this maximal operator was studied by Dziubański et al. [9] (see also [11, 25])

As for the (vector-valued) dual Riesz transform $\mathscr{R}_{\mathscr{L}}^{*}$ defined above, we have the following estimate given in [21] (see also [19]).

Theorem 11. Let $V \in \mathscr{B}_{q}$ with $q \in[Q / 2, Q)$, and let $p_{0}$ be a number such that $1 / p_{0}=1 / q-1 / Q$. Then, the (vector-valued) dual Riesz transform $\mathscr{R}_{\mathscr{L}}^{*}$ is bounded on $L^{p}\left(\mathbb{H}^{n}\right)$ for $\left(p_{0}\right)^{\prime}<$ $p<\infty$.

By duality, we could obtain the following result.

Theorem 12. Let $V \in \mathscr{B}_{q}$ with $q \in[Q / 2, Q)$, and let $p_{0}$ be a number such that $1 / p_{0}=1 / q-1 / Q$. Then, the (vector-valued) Riesz transform $\mathscr{R}_{\mathscr{L}}$ is bounded on $L^{p}\left(\mathbb{H}^{n}\right)$ for $1<p<p_{0}$.

Moreover, it will be proved in Section 4 that $\mathscr{R}_{\mathscr{L}}$ is of weak-type $(1,1)$ on the Heisenberg group. The case where $q \in[Q, \infty)$ is also considered in Section 4.

Remark 13.

(i) It can be shown that the range of $p$ in the above theorems is optimal (see [3]). In this paper, the authors also proved that the dual Riesz transform $\mathscr{R}_{\mathscr{L}}^{*}$ is bounded on $\mathrm{BMO}_{\mathscr{L}}\left(\mathbb{H}^{n}\right)$ and gave the FeffermanStein-type decomposition of $\mathrm{BMO}_{\mathscr{L}}$ functions with respect to $\mathscr{R}_{j}^{*}, j=1,2, \cdots, 2 n$

(ii) It was shown in [26] that when $V \equiv 0$, the operators $\mathscr{R}:=\nabla_{\mathbb{H}^{n}}\left(-\Delta_{\mathbb{H}^{n}}\right)^{-1 / 2}$ and $\mathscr{R}^{*}=\left(-\Delta_{\mathbb{H}^{n}}\right)^{-1 / 2} \nabla_{\mathbb{H}^{n}}$ are uniformly bounded on $L^{p}\left(\mathbb{H}^{n}\right)$ with respect to $n$. More specifically, for every $1<p<\infty$, there exists a constant $C_{p}>0$ such that for every $n \in \mathbb{N}$

$$
\|\mathscr{R}(f)\|_{L^{p}\left(\mathbb{H}^{n}\right)}+\left\|\mathscr{R}^{*}(f)\right\|_{L^{p}\left(\mathbb{H}^{n}\right)} \leq C_{p}\|f\|_{L^{p}\left(\mathbb{H}^{n}\right)}
$$

(iii) Recall that in the setting of $\mathbb{R}^{n}$, the Riesz transform and its dual form were originally studied by Shen in [10]. It can be proved that the analog of Theorem 11 (also Theorem 12) on the Euclidean space is also true by the same argument (see [10,27]). For the corresponding estimates for commutators generated by BMO functions, the reader is referred to $[11,12,28]$ for more details

The paper is organized as follows. In Section 2, we introduce the Morrey space and weak Morrey space associated with the Schrödinger operator $\mathscr{L}$ on $\mathbb{H}^{n}$ and state our main results: Theorems 18, 19, 22, 23, and 24. Section 3 is devoted to Proofs of Theorems 18 and 19, which establish the strongtype and weak-type estimates for the semigroup maximal function $\mathscr{T}_{\mathscr{L}}^{*}$ in the framework of Morrey spaces. The corresponding estimates for some other maximal functions are also proved in this section. Section 4 is devoted to proving the boundedness properties of the Riesz transform $\mathscr{R}_{\mathscr{L}}$ and its dual form $\mathscr{R}_{\mathscr{L}}^{*}$. In Section 5, we extend the above results to the generalized Morrey spaces.

Throughout this paper, $C>0$ denotes a universal constant which may change from line to line, and a subscript is added when we wish to make clear its dependence on the parameter in the subscript. The notation $\mathbf{X} \lesssim \mathbf{Y}$ means that $\mathbf{X} \leq C \mathbf{Y}$ for some positive constant $C$. If $\mathbf{X} \leqslant \mathbf{Y}$ and $\mathbf{Y} \leqslant \mathbf{X}$, then we write $\mathbf{X} \approx \mathbf{Y}$ to denote the equivalence of $\mathbf{X}$ and $\mathbf{Y}$. For any $p \in[1, \infty)$, the notation $p^{\prime}$ denotes its conjugate number, namely, $1 / p+1 / p^{\prime}=1$ and $1^{\prime}=\infty$.

\section{Definitions and Main Theorems}

A few historic remarks are in order. The classical Morrey space $M^{p, \lambda}\left(\mathbb{R}^{n}\right)$ was originally introduced and studied by Morrey in [29] to deal with the local behavior of solutions to second-order elliptic partial differential equations. Since then, this space was systematically developed by a number of authors. Nowadays, this space has been investigated extensively and widely used in analysis, geometry, mathematical physics, and other related fields. We denote by $M^{p, \lambda}\left(\mathbb{R}^{n}\right)$ the Morrey space, which consists of all $p$-locally integrable functions $f$ on $\mathbb{R}^{n}$ such that

$$
\begin{aligned}
\|f\|_{M^{p, \lambda}\left(\mathbb{R}^{n}\right)} & :=\sup _{x \in \mathbb{R}^{n}, r>0} r^{-\lambda / p}\|f\|_{L^{p}(B(x, r))} \\
& =\sup _{x \in \mathbb{R}^{n}, r>0} r^{-\lambda / p}\left(\int_{B(x, r)}|f(y)|^{p} d y\right)^{1 / p}<\infty,
\end{aligned}
$$

where $1 \leq p<\infty$ and $0 \leq \lambda \leq n$. It is known that $M^{p, \lambda}\left(\mathbb{R}^{n}\right)$ is an extension of $L^{p}\left(\mathbb{R}^{n}\right)$ in the sense that $M^{p, 0}\left(\mathbb{R}^{n}\right)=L^{p}\left(\mathbb{R}^{n}\right)$. Note that $M^{p, n}\left(\mathbb{R}^{n}\right)=L^{\infty}\left(\mathbb{R}^{n}\right)$ by the Lebesgue differentiation theorem. If $\lambda<0$ or $\lambda>n$, then $M^{p, \lambda}\left(\mathbb{R}^{n}\right)=\Theta$, where $\Theta$ is the set of all functions equivalent to 0 on $\mathbb{R}^{n}$. We also denote by $W M^{1, \lambda}\left(\mathbb{R}^{n}\right)$ the weak Morrey space, which consists of all measurable functions $f$ on $\mathbb{R}^{n}$ such that

$$
\begin{aligned}
\|f\|_{W M^{1, \lambda}\left(\mathbb{R}^{n}\right)} & =\sup _{x \in \mathbb{R}^{n}, r>0} r^{-\lambda}\|f\|_{W L^{1}(B(x, r))} \\
& =\sup _{x \in \mathbb{R}^{n}, r>0} r^{-\lambda} \sup _{\sigma>0} \sigma|\{y \in B(x, r):|f(y)|>\sigma\}|<\infty .
\end{aligned}
$$


For the properties of classical Morrey spaces, we refer the readers to [30-34] and the references therein. Moreover, the Morrey spaces were found to have many important applications to the Navier-Stokes equations, the Schrödinger equations, the elliptic equations with discontinuous coefficients, and the potential analysis (one can see [30, 35-38]).

In this section, we introduce some types of Morrey spaces associated with the Schrödinger operator $\mathscr{L}$ on $\mathbb{H}^{n}$ (see [39]) and then give our main results.

Definition 14. Let $\rho$ be the auxiliary function determined by $V \in \mathscr{B}_{q}$ with $q \in[Q / 2, \infty)$. Let $1 \leq p<\infty$ and $0 \leq \kappa<1$. For each given $0<\theta<\infty$, the Morrey space $L_{\rho, \theta}^{p, \kappa}\left(\mathbb{H}^{n}\right)$ is defined to be the set of all $p$-locally integrable functions $f$ on $\mathbb{H}^{n}$ such that

$$
\left(\frac{1}{\left|B\left(u_{0}, r\right)\right|^{\kappa}} \int_{B\left(u_{0}, r\right)}|f(u)|^{p} d u\right)^{1 / p} \leq C \cdot\left[1+\frac{r}{\rho\left(u_{0}\right)}\right]^{\theta}
$$

holds for every ball $B\left(u_{0}, r\right)$ in $\mathbb{H}^{n}$, where $u_{0}$ and $r$ denote the center and radius of $B\left(u_{0}, r\right)$, respectively. The smallest constant appearing in (40) is called the norm of $f$, which is denoted by $\|f\|_{L_{\rho \theta}^{p, \kappa}\left(\mathbb{H}^{n}\right)}$. It is a Banach space with respect to the norm $\|\cdot\|_{L_{\rho, \theta}^{p, \kappa}\left(\mathbb{H}^{n}\right)}$. Define

$$
L_{\rho, \infty}^{p, \kappa}\left(\mathbb{H}^{n}\right):=\bigcup_{0<\theta<\infty} L_{\rho, \theta}^{p, \kappa}\left(\mathbb{H}^{n}\right) .
$$

Definition 15. Let $\rho$ be the auxiliary function determined by $V \in \mathscr{B}_{q}$ with $q \in[Q / 2, \infty)$. Let $p=1$ and $0 \leq \kappa<1$. For each given $0<\theta<\infty$, the weak Morrey space $W L_{\rho, \theta}^{1, \kappa}\left(\mathbb{H}^{n}\right)$ is defined to be the set of all measurable functions $f$ on $\mathbb{H}^{n}$ such that

$\frac{1}{\left|B\left(u_{0}, r\right)\right|^{\kappa}} \sup _{\lambda>0} \lambda \cdot\left|\left\{u \in B\left(u_{0}, r\right):|f(u)|>\lambda\right\}\right| \leq C \cdot\left[1+\frac{r}{\rho\left(u_{0}\right)}\right]^{\theta}$,

holds for every ball $B\left(u_{0}, r\right)$ in $\mathbb{H}^{n}$. The smallest constant appearing in (42) is called the (quasi-)norm of $f$, which is denoted by $\|f\|_{W L_{\rho, \theta}^{1, \kappa}\left(\mathbb{H}^{n}\right)}$. It is a (quasi-)Banach space with respect to the (quasi-)norm $\|\cdot\|_{W L_{\rho, \theta}^{1, \kappa}\left(\mathbb{H}^{n}\right)}$. Correspondingly, we define

$$
W L_{\rho, \infty}^{1, \kappa}\left(\mathbb{H}^{n}\right):=\bigcup_{0<\theta<\infty} W L_{\rho, \theta}^{1, \kappa}\left(\mathbb{H}^{n}\right)
$$

\section{Remark 16.}

(i) Obviously, if we take $\theta=0$ or $V \equiv 0$, then this Morrey space $L_{\rho, \theta}^{p, \kappa}\left(\mathbb{H}^{n}\right)$ (or weak Morrey space $W L_{\rho, \theta}^{1, \kappa}\left(\mathbb{H}^{n}\right)$ ) is just the Morrey space $L^{p, \kappa}\left(\mathbb{H}^{n}\right)$ (or weak Morrey space $\left.W L^{1, \kappa}\left(\mathbb{H}^{n}\right)\right)$, which was defined and studied by Guliyev et al. [40] (ii) According to the above definitions, one has

$$
\begin{gathered}
L^{p, \kappa}\left(\mathbb{H}^{n}\right) \subset L_{\rho, \theta_{1}}^{p, \kappa}\left(\mathbb{H}^{n}\right) \subset L_{\rho, \theta_{2}}^{p, \kappa}\left(\mathbb{H}^{n}\right), \\
W L^{1, \kappa}\left(\mathbb{H}^{n}\right) \subset W L_{\rho, \theta_{1}}^{1, \kappa}\left(\mathbb{H}^{n}\right) \subset W L_{\rho, \theta_{2}}^{1, \kappa}\left(\mathbb{H}^{n}\right),
\end{gathered}
$$

whenever $0<\theta_{1}<\theta_{2}<\infty$. Hence,

$$
\begin{gathered}
L^{p, \kappa}\left(\mathbb{H}^{n}\right) \subset L_{\rho, \infty}^{p, \kappa}\left(\mathbb{H}^{n}\right), \\
W L^{1, \kappa}\left(\mathbb{H}^{n}\right) \subset W L_{\rho, \infty}^{1, \kappa}\left(\mathbb{H}^{n}\right),
\end{gathered}
$$

for all $(p, \kappa) \in[1, \infty) \times[0,1)$. When $\kappa=0$, the spaces $L^{p, \kappa}$ $\left(\mathbb{H}^{n}\right)$ and $W L^{1, \kappa}\left(\mathbb{H}^{n}\right)$ reduce to $L^{p}\left(\mathbb{H}^{n}\right)$ and $W L^{1}\left(\mathbb{H}^{n}\right)$, respectively.

(iii) It follows directly from Chebyshev's inequality that $\|f\|_{W L_{\rho, \theta}^{1, \kappa}\left(\mathbb{H}^{n}\right)} \leq\|f\|_{L_{\rho, \theta}^{1, \kappa}\left(\mathbb{H}^{n}\right)}$, and hence,

$$
\begin{gathered}
L_{\rho, \theta}^{1, \kappa}\left(\mathbb{H}^{n}\right) \subset W L_{\rho, \theta}^{1, \kappa}\left(\mathbb{H}^{n}\right), \\
L_{\rho, \infty}^{1, \kappa}\left(\mathbb{H}^{n}\right) \subset W L_{\rho, \infty}^{1, \kappa}\left(\mathbb{H}^{n}\right) .
\end{gathered}
$$

Moreover, the inclusion is strict.

\section{Remark 17.}

(i) We can define a norm on the space $L_{\rho, \infty}^{p, \kappa}\left(\mathbb{H}^{n}\right)$ (see [39]), which makes it into a Banach space. In view of (44), for any given $f \in L_{\rho, \infty}^{p, \kappa}\left(\mathbb{H}^{n}\right)$, let

$$
\theta^{*}:=\inf \left\{\theta>0: f \in L_{\rho, \theta}^{p, \kappa}\left(\mathbb{H}^{n}\right)\right\}
$$

Now define the functional $\|\cdot\|_{a}$ by

$$
\|f\|_{\star}=\|f\|_{L_{\rho, \infty}^{p, \kappa}\left(\mathbb{H}^{n}\right)}:=\|f\|_{L_{\rho, \theta^{*}}^{p, \kappa}\left(\mathbb{H}^{n}\right)} .
$$

It is easy to check that the functional $\|\cdot\|_{\star}$ defined by (49) is indeed a norm on $L_{\rho, \infty}^{p, \kappa}\left(\mathbb{H}^{n}\right)$ provided $(p, \kappa) \in[1, \infty) \times$ $(0,1)$; i.e., it satisfies the following conditions:

(a) It is positive definite: $\|f\|_{\star} \geq 0$, and $\|f\|_{\star}=0$ if and only if $f=0$

(b) It is multiplicative: $\|\lambda f\|_{\star}=|\lambda|\|f\|_{\star}$, for any $\lambda \in \mathbb{R}$

(c) It satisfies the triangle inequality: $\|f+g\|_{\star} \leq\|f\|_{\star}+$ $\|g\|_{\star}$, for any $f, g \in L_{\rho, \infty}^{p, \kappa}\left(\mathbb{H}^{n}\right)$

(ii) In view of (45), for any given $f \in W L_{\rho, \infty}^{1, \kappa}\left(\mathbb{H}^{n}\right)$, let

$$
\theta^{* *}:=\inf \left\{\theta>0: f \in W L_{\rho, \theta}^{1, \kappa}\left(\mathbb{H}^{n}\right)\right\}
$$


Similarly, we define the functional $\|\cdot\|_{\star \star}$ by

$$
\|f\|_{\star \star}=\|f\|_{W L_{\rho, \infty}^{1, \kappa}\left(\mathbb{H}^{n}\right)}:=\|f\|_{W L_{\rho, \theta^{* *}}^{1, \kappa}\left(\mathbb{H}^{n}\right)} .
$$

It is easily checked that the functional $\|\cdot\|_{\star \star}$ defined by (51) is a (quasi-)norm on $W L_{\rho, \infty}^{1, \kappa}\left(\mathbb{H}^{n}\right)$ for all $0<\kappa<1$; i.e., it satisfies the following conditions:

(a) It is positive definite: $\|f\|_{\star \star} \geq 0$, and $\|f\|_{\star \star}=0$ if and only if $f=0$

(b) It is multiplicative: $\|\lambda f\|_{\star \star}=|\lambda|\|f\|_{\star \star}$, for any $\lambda \in \mathbb{R}$

(c) It satisfies the inequality: $\|f+g\|_{\star \star} \leq 2\left(\|f\|_{\star \star}+\right.$ $\left.\|g\|_{\star \star}\right)$, for any $f, g \in W L_{\rho, \infty}^{1, \kappa}\left(\mathbb{H}^{n}\right)$

The space $W L_{\rho, \infty}^{1, \kappa}\left(\mathbb{H}^{n}\right)$ is a (quasi-)Banach space with respect to the (quasi-)norm $\|\cdot\|_{\star \star}$.

Since Morrey space $L_{\rho, \theta}^{p, \kappa}\left(\mathbb{H}^{n}\right)$ (or weak Morrey space $W$ $\left.L_{\rho, \theta}^{1, \kappa}\left(\mathbb{H}^{n}\right)\right)$ could be viewed as an extension of the Lebesgue space (or the weak Lebesgue space) on $\mathbb{H}^{n}$ (when $\kappa=\theta=0$, or $\kappa=0, V \equiv 0$ ), it is natural to study the boundedness properties of the operators $\mathscr{T}_{\mathscr{L}}^{*}, \mathscr{R}_{\mathscr{L}}$, and $\mathscr{R}_{\mathscr{L}}^{*}$ in the context of Morrey spaces. In this paper, we will extend Theorems 9, 11 , and 12 to the Morrey spaces on $\mathbb{H}^{n}$. Let $\rho$ be the same as before. Now let us formulate our main results as follows.

Theorem 18. Let $1<p<\infty, 0<\kappa<1$, and $0<\theta<\infty$. If $V$ $\in \mathscr{B}_{q}$ with $q \in[Q / 2, \infty)$, then the semigroup maximal function $\mathscr{T}_{\mathscr{L}}^{*}$ is a bounded sublinear operator on $L_{\rho, \theta}^{p, \kappa}\left(\mathbb{H}^{n}\right)$.

Theorem 19. Let $p=1,0<\kappa<1$, and $0<\theta<\infty$. If $V \in \mathscr{B}_{q}$ with $q \in[Q / 2, \infty)$, then the semigroup maximal function $\mathscr{T}_{\mathscr{L}}^{*}$ is a bounded sublinear operator from $L_{\rho, \theta}^{1, \kappa}\left(\mathbb{H}^{n}\right)$ into $W L_{\rho, \theta}^{1, \kappa}\left(\mathbb{H}^{n}\right)$.

As an immediate consequence of Theorems 18 and 19 and Remark 17, we have the following results.

Corollary 20. Let $1<p<\infty$ and $0<\kappa<1$. If $V \in \mathscr{B}_{q}$ with $q$ $\in[Q / 2, \infty)$, then the semigroup maximal function $\mathscr{T}_{\mathscr{L}}^{*}$ is a bounded sublinear operator on $L_{\rho, \infty}^{p, \kappa}\left(\mathbb{H}^{n}\right)$.

Corollary 21. Let $p=1$ and $0<\kappa<1$. If $V \in \mathscr{B}_{q}$ with $q \in[Q /$ $2, \infty)$, then the semigroup maximal function $\mathscr{T}_{\mathscr{L}}^{*}$ is a bounded sublinear operator from $L_{\rho, \infty}^{1, \kappa}\left(\mathbb{H}^{n}\right)$ into $W L_{\rho, \infty}^{1, \kappa}\left(\mathbb{H}^{n}\right)$.

Theorem 22. Let $0<\kappa<1$ and $0<\theta<\infty$. If $V \in \mathscr{B}_{q}$ with $q$ $\in[Q / 2, Q)$, and $p_{0}$ is a number such that $1 / p_{0}=1 / q-1 / Q$, then the dual Riesz transform $\mathscr{R}_{\mathscr{L}}^{*}$ is a bounded linear operator on $L_{\rho, \theta}^{p, \kappa}\left(\mathbb{H}^{n}\right)$ provided that $\left(p_{0}\right)^{\prime}<p<\infty$.
Theorem 23. Let $0<\theta<\infty$. If $V \in \mathscr{B}_{q}$ with $q \in[Q / 2, Q)$, and $p_{0}$ is a number such that $1 / p_{0}=1 / q-1 / Q$, then the Riesz transform $\mathscr{R}_{\mathscr{L}}$ is a bounded linear operator on $L_{\rho, \theta}^{p, \kappa}\left(\mathbb{H}^{n}\right)$ provided that $1<p<p_{0}$ and $0<\kappa<1 / s^{\prime}$ with $s:=p_{0} / p$.

It is worth pointing out that we cannot use Theorem 22 to prove Theorem 23 in a direct way by duality, since the predual to $L_{\rho, \theta}^{p, \kappa}\left(\mathbb{H}^{n}\right)$ is unknown. Motivated by the ideas in $[30,31]$, it is an interesting and natural problem to investigate the dual theory for the Morrey space $L_{\rho, \theta}^{p, \kappa}\left(\mathbb{H}^{n}\right)$ through a geometric analysis of the Hausdorff capacity and Choquet integrals, which will be treated in a subsequent paper. In addition, we will prove that the operator $\mathscr{R}_{\mathscr{L}}$ is of weaktype $(1,1)$. Based on this result, we can further prove the following.

Theorem 24. Let $0<\theta<\infty$. If $V \in \mathscr{B}_{q}$ with $q \in[Q / 2, Q)$, and $p_{0}$ is a number such that $1 / p_{0}=1 / q-1 / Q$, then the Riesz transform $\mathscr{R}_{\mathscr{L}}$ is a bounded linear operator from $L_{\rho, \theta}^{1, \kappa}\left(\mathbb{H}^{n}\right)$ into $W L_{\rho, \theta}^{1, \kappa}\left(\mathbb{H}^{n}\right)$ provided that $0<\kappa<1 /\left(p_{0}\right)^{\prime}$.

As a straightforward consequence of Theorems 22-24 and Remark 17, we obtain the following estimates.

Corollary 25. Let $0<\kappa<1$. If $V \in \mathscr{B}_{q}$ with $q \in[Q / 2, Q)$, and $p_{0}$ is a number such that $1 / p_{0}=1 / q-1 / Q$, then the dual Riesz transform $\mathscr{R}_{\mathscr{L}}^{*}$ is a bounded linear operator on $L_{\rho, \infty}^{p, \kappa}\left(\mathbb{H}^{n}\right)$ provided that $\left(p_{0}\right)^{\prime}<p<\infty$.

Corollary 26. If $V \in \mathscr{B}_{q}$ with $q \in[Q / 2, Q)$, and $p_{0}$ is a number such that $1 / p_{0}=1 / q-1 / Q$, then the Riesz transform $\mathscr{R}_{\mathscr{L}}$ is a bounded linear operator on $L_{\rho, \infty}^{p, \kappa}\left(\mathbb{H}^{n}\right)$ provided that $1<p<$ $p_{0}$ and $0<\kappa<1 / s^{\prime}$ with $s:=p_{0} / p$.

Corollary 27. If $V \in \mathscr{B}_{q}$ with $q \in[Q / 2, Q)$, and $p_{0}$ is a number such that $1 / p_{0}=1 / q-1 / Q$, then the Riesz transform $\mathscr{R}_{\mathscr{L}}$ is a bounded linear operator from $L_{\rho, \infty}^{1, \kappa}\left(\mathbb{H}^{n}\right)$ into $W L_{\rho, \infty}^{1, \kappa}\left(\mathbb{H}^{n}\right)$ provided that $0<\kappa<1 /\left(p_{0}\right)^{\prime}$.

\section{Boundedness of the Semigroup Maximal Functions}

In this section, we will prove the conclusions of Theorems 18 and 19. Let $\mathscr{P}_{t}(u, v)$ denote the integral kernel of $e^{-t \mathscr{L}}$ related to the Schrödinger operator $\mathscr{L}$ (see [3]). Then, we can write $\mathscr{T}_{\mathscr{L}}^{*}$ as follows:

$\mathscr{T}_{\mathscr{L}}^{*}(f)(u)=\sup _{t>0}\left|e^{-t \mathscr{L}} f(u)\right|=\sup _{t>0}\left|\int_{\mathbb{H}^{n}} \mathscr{P}_{t}(u, v) f(v) d v\right|, u \in \mathbb{H}^{n}$.

Proof of Theorem 18. For any given $f \in L_{\rho, \theta}^{p, \kappa}\left(\mathbb{H}^{n}\right)$ with $(p, \kappa)$ $\epsilon(1, \infty) \times(0,1)$ and $\theta \in(0, \infty)$, by definition, we only need to show that for any given ball $B=B\left(u_{0}, r\right)$ of $\mathbb{H}^{n}$, the 
following inequality

$$
\left(\frac{1}{\left|B\left(u_{0}, r\right)\right|^{\kappa}} \int_{B\left(u_{0}, r\right)}\left|\mathscr{T}_{\mathscr{L}}^{*}(f)(u)\right|^{p} d u\right)^{1 / p} \lesssim\left[1+\frac{r}{\rho\left(u_{0}\right)}\right]^{\theta},
$$

holds true. By a standard argument, we decompose the function $f$ as

$$
\left\{\begin{array}{l}
f=f_{1}+f_{2} \in L_{\rho, \theta}^{p, \kappa}\left(\mathbb{H}^{n}\right), \\
f_{1}=f \cdot \chi_{2 B}, \\
f_{2}=f \cdot \chi_{(2 B)} c
\end{array}\right.
$$

where $2 B$ denotes the open ball centered at $u_{0}$ of radius $2 r, \chi_{E}$ denotes the characteristic function of the set $E$, and $(2 B)^{\complement}=$ $\mathbb{H}^{n} \backslash(2 B)$ denotes its complement. Then, by the sublinearity of $\mathscr{T}_{\mathscr{L}}^{*}$, we write

$$
\begin{aligned}
& \left(\frac{1}{\left|B\left(u_{0}, r\right)\right|^{\kappa}} \int_{B\left(u_{0}, r\right)}\left|\mathscr{T}_{\mathscr{L}}^{*}(f)(u)\right|^{p} d u\right)^{1 / p} \\
& \leq\left(\frac{1}{\left|B\left(u_{0}, r\right)\right|^{\kappa}} \int_{B\left(u_{0}, r\right)}\left|\mathscr{T}_{\mathscr{L}}^{*}\left(f_{1}\right)(u)\right|^{p} d u\right)^{1 / p} \\
& \quad+\left(\frac{1}{\left|B\left(u_{0}, r\right)\right|^{\kappa}} \int_{B\left(u_{0}, r\right)}\left|\mathscr{T}_{\mathscr{L}}^{*}\left(f_{2}\right)(u)\right|^{p} d u\right)^{1 / p}:=I_{1}+I_{2} .
\end{aligned}
$$

Let us consider the first term $I_{1}$. Making use of the first part of Theorem 9, we have

$$
\begin{aligned}
I_{1} & =\left(\frac{1}{\left|B\left(u_{0}, r\right)\right|^{\kappa}} \int_{B\left(u_{0}, r\right)}\left|\mathscr{T}_{\mathscr{L}}^{*}(f)(u)\right|^{p} d u\right)^{1 / p} \\
& \leq C \cdot \frac{1}{|B|^{\kappa / p}}\left(\int_{\mathbb{H}^{n}} \| f_{1}(u)^{p} d u\right)^{1 / p} \\
& =C \cdot \frac{1}{|B|^{\kappa / p}}\left(\int_{2 B}|f(u)|^{p} d u\right)^{1 / p} \\
& \leq C\|f\|_{L_{\rho, \theta}^{p, \kappa}\left(\mathbb{H}^{n}\right)} \cdot \frac{|2 B|^{\kappa / p}}{|B|^{\kappa / p}} \cdot\left[1+\frac{2 r}{\rho\left(u_{0}\right)}\right]^{\theta} .
\end{aligned}
$$

Moreover, note that for any fixed $\theta \in(0, \infty)$,

$$
1 \leq\left[1+\frac{2 r}{\rho\left(u_{0}\right)}\right]^{\theta} \leq 2^{\theta}\left[1+\frac{r}{\rho\left(u_{0}\right)}\right]^{\theta} .
$$

This, combined with (10), yields

$$
I_{1} \leq C_{\theta, n}\|f\|_{L_{\rho, \theta}^{p, \kappa}\left(\mathbb{H}^{n}\right)}\left[1+\frac{r}{\rho\left(u_{0}\right)}\right]^{\theta} .
$$

We now turn to estimate the second term $I_{2}$. We first assert that the following inequality

$$
\mathscr{T}_{\mathscr{L}}^{*}\left(f_{2}\right)(u) \leq C_{N, A} \int_{(2 B)^{\complement}}\left[1+\frac{\left|v^{-1} u\right|}{\rho(u)}\right]^{-N} \frac{1}{\left|v^{-1} u\right|^{Q}} \bullet|f(v)| d v,
$$

holds for any $u \in B\left(u_{0}, r\right)$, where $N$ and $A$ are given as in Lemma 7. Indeed, this can be done by considering the following two cases: $0<t \leq\left|v^{-1} u\right|^{2}$ and $\left|v^{-1} u\right|^{2}<t<\infty$. It follows directly from Lemma 7 that

$$
\begin{aligned}
\mathscr{T}_{\mathscr{L}}^{*}\left(f_{2}\right)(u)= & \sup _{t>0}\left|\int_{\mathbb{H}^{n}} \mathscr{P}_{t}(u, v) f_{2}(v) d v\right| \\
\leq & \sup _{t>0} \mid \int_{\mathbb{H}^{n}} \frac{C_{N}}{t^{Q / 2}} \cdot \exp \left(-\frac{\left|v^{-1} u\right|^{2}}{A t}\right) \\
& \cdot\left[1+\frac{\sqrt{t}}{\rho(u)}\right]^{-N}\left|f_{2}(v)\right| d v \mid \\
\leq & \sup _{\left|v^{-1} u\right|^{2}<t<\infty} \mid \int_{\mathbb{H}^{n}} \frac{C_{N}}{t^{Q / 2}} \cdot \exp \left(-\frac{\left|v^{-1} u\right|^{2}}{A t}\right) \\
& \cdot\left[1+\frac{\sqrt{t}}{\rho(u)}\right]^{-N}\left|f_{2}(v)\right| d v\left|+\sup _{0<t \leq\left|v^{-1} u\right|^{2}}\right| \int_{\mathbb{H}^{n}} \frac{C_{N}}{t^{Q / 2}} \\
& \cdot \exp \left(-\frac{\left|v^{-1} u\right|^{2}}{A t}\right)\left[1+\frac{\sqrt{t}}{\rho(u)}\right]^{-N}\left|f_{2}(v)\right| d v \mid \\
:= & \mathscr{T}_{\infty}^{*}\left(f_{2}\right)(u)+\mathscr{T}_{0}^{*}\left(f_{2}\right)(u) .
\end{aligned}
$$

When $t>\left|v^{-1} u\right|^{2}$, then $\sqrt{t}>\left|v^{-1} u\right|$, and hence,

$$
\begin{aligned}
\mathscr{T}_{\infty}^{*}\left(f_{2}\right)(u) \leq & \sup _{\left|v^{-1} u\right|^{2}<t<\infty} \int_{\mathbb{H}^{n}} \frac{C_{N, A}}{t^{Q / 2}} \cdot\left(\frac{\left|v^{-1} u\right|^{2}}{t}\right)^{-Q / 2}\left[1+\frac{\sqrt{t}}{\rho(u)}\right]^{-N} \\
& \cdot\left|f_{2}(v)\right| d v \\
= & \sup _{\left|v^{-1} u\right|^{2}<t<\infty}\left|\int_{\mathbb{H}^{n}} \frac{C_{N, A}}{\left|v^{-1} u\right|^{Q}} \cdot\left[1+\frac{\sqrt{t}}{\rho(u)}\right]^{-N}\right| f_{2}(v)|d v| \\
\leq & C_{N, A} \int_{(2 B)^{\complement}}\left[1+\frac{\left|v^{-1} u\right|}{\rho(u)}\right]^{-N} \frac{1}{\left|v^{-1} u\right|^{Q}} \cdot|f(v)| d v .
\end{aligned}
$$


On the other hand, we can easily see that

$$
\begin{aligned}
\mathscr{T}_{0}^{*}\left(f_{2}\right)(u) \leq & \sup _{0<t \leq\left|v^{-1} u\right|^{2}} \int_{\mathbb{H}^{n}} \frac{C_{N, A}}{t^{Q / 2}} \cdot\left(\frac{\left|v^{-1} u\right|^{2}}{t}\right)^{-(\mathrm{Q} / 2+N / 2)} \\
& \cdot\left[1+\frac{\sqrt{t}}{\rho(u)}\right]^{-N}\left|f_{2}(v)\right| d v \\
= & \sup _{0<t \leq\left|v^{-1} u\right|^{2}} \int_{\mathbb{H}^{n}} \frac{C_{N, A}}{\left|v^{-1} u\right|^{Q}} \cdot\left(\frac{\sqrt{t}}{\left|v^{-1} u\right|}\right)^{N}\left[1+\frac{\sqrt{t}}{\rho(u)}\right]^{-N} \\
& \cdot\left|f_{2}(v)\right| d v .
\end{aligned}
$$

When $0<t \leq\left|v^{-1} u\right|^{2}$, then $\sqrt{t} \leq\left|v^{-1} u\right|$. In this case, it is easy to check that for any $N \in \mathbb{N}$,

$$
\left(\frac{\sqrt{t}}{\left|v^{-1} u\right|}\right)^{N} \leq\left[\frac{\sqrt{t}+\rho(u)}{\left|v^{-1} u\right|+\rho(u)}\right]^{N} .
$$

From this, it follows immediately that

$$
\begin{aligned}
\mathscr{T}_{0}^{*}\left(f_{2}\right)(u) \leq & \sup _{0<t \leq\left|v^{-1} u\right|^{2}} \int_{\mathbb{H}^{n}} \frac{C_{N, A}}{\left|v^{-1} u\right|^{Q}} \cdot\left[\frac{\sqrt{t}+\rho(u)}{\left|v^{-1} u\right|+\rho(u)}\right]^{N} \\
& \cdot\left[\frac{\sqrt{t}+\rho(u)}{\rho(u)}\right]^{-N}\left|f_{2}(v)\right| d v \\
\leq & C_{N, A} \int_{(2 B)^{\complement}}\left[1+\frac{\left|v^{-1} u\right|}{\rho(u)}\right]^{-N} \frac{1}{\left|v^{-1} u\right|^{Q}} \cdot|f(v)| d v .
\end{aligned}
$$

Putting all together produces the required inequality (59). Notice that for any $u \in B\left(u_{0}, r\right)$ and $v \in(2 B)^{\complement}$, one has

$$
\begin{aligned}
& \left|v^{-1} u\right|=\left|\left(v^{-1} u_{0}\right) \cdot\left(u_{0}^{-1} u\right)\right| \leq\left|v^{-1} u_{0}\right|+\left|u_{0}^{-1} u\right|, \\
& \left|v^{-1} u\right|=\left|\left(v^{-1} u_{0}\right) \cdot\left(u_{0}^{-1} u\right)\right| \geq\left|v^{-1} u_{0}\right|-\left|u_{0}^{-1} u\right| .
\end{aligned}
$$

Thus,

$$
\frac{1}{2}\left|v^{-1} u_{0}\right| \leq\left|v^{-1} u\right| \leq \frac{3}{2}\left|v^{-1} u_{0}\right| .
$$

That is, $\left|v^{-1} u\right| \approx\left|v^{-1} u_{0}\right|$. Combining this fact with (59) yields that for any $u \in B\left(u_{0}, r\right)$ and any positive integer $N \in \mathbb{N}$,

$$
\begin{aligned}
\mathscr{T}_{\mathscr{L}}^{*}\left(f_{2}\right)(u) \leq & C_{N, A, n} \int_{(2 B)^{\complement}}\left[1+\frac{\left|v^{-1} u_{0}\right|}{\rho(u)}\right]^{-N} \frac{1}{\left|\mathrm{v}^{-1} \mathrm{u}_{0}\right|^{Q}} \cdot|f(v)| d v \\
= & C_{N, A, n} \sum_{k=1}^{\infty} \int_{2^{k} r \leq\left|v^{-1} u_{0}\right|<2^{k+1} r}\left[1+\frac{\left|v^{-1} u_{0}\right|}{\rho(u)}\right]^{-N} \\
& \cdot \frac{1}{\left|v^{-1} u_{0}\right|^{Q}} \cdot|f(v)| d v \\
\leq & C_{N, A, n} \sum_{k=1}^{\infty} \frac{1}{\left|B\left(u_{0}, 2^{k+1} r\right)\right|} \int_{\left|v^{-1} u_{0}\right|<2^{k+1} r} \\
& \cdot\left[1+\frac{2^{k} r}{\rho(u)}\right]^{-N}|f(v)| d v .
\end{aligned}
$$

Furthermore, in view of (21) and (57), we can see that the above expression (67) does not exceed

$$
\begin{aligned}
C & \sum_{k=1}^{\infty} \frac{1}{\left|B\left(u_{0}, 2^{k+1} r\right)\right|} \int_{B\left(u_{0}, 2^{k+1} r\right)}\left[1+\frac{r}{\rho\left(u_{0}\right)}\right]^{N \cdot\left(N_{0} /\left(N_{0}+1\right)\right)} \\
& \times\left[1+\frac{2^{k} r}{\rho\left(u_{0}\right)}\right]^{-N}|f(v)| d v \\
\leq & C \sum_{k=1}^{\infty}\left[1+\frac{2^{k} r}{\rho\left(u_{0}\right)}\right]^{-N \cdot\left(1 /\left(N_{0}+1\right)\right)} \frac{1}{\left|B\left(u_{0}, 2^{k+1} r\right)\right|} \int_{B\left(u_{0}, 2^{k+1} r\right)}|f(v)| d v \\
\leq & C \sum_{k=1}^{\infty}\left[1+\frac{2^{k+1} r}{\rho\left(u_{0}\right)}\right]^{-N \cdot\left(1 /\left(N_{0}+1\right)\right)} \frac{1}{\left|B\left(u_{0}, 2^{k+1} r\right)\right|} \int_{B\left(u_{0}, 2^{k+1} r\right)}|f(v)| d v .
\end{aligned}
$$

By using the Hölder inequality, we obtain that for each fixed $k \in \mathbb{N}$,

$$
\begin{aligned}
& \frac{1}{\left|B\left(u_{0}, 2^{k+1} r\right)\right|} \int_{B\left(u_{0}, 2^{k+1} r\right)}|f(v)| d v \\
& \leq \frac{1}{\left|B\left(u_{0}, 2^{k+1} r\right)\right|}\left(\int_{B\left(u_{0}, 2^{k+1} r\right)}|f(v)|^{p} d v\right)^{1 / p}\left(\int_{B\left(u_{0}, 2^{k+1} r\right)} 1 d v\right)^{1 / p^{\prime}} \\
& \leq C\|f\|_{L_{\rho, \theta}^{p, k}\left(\mathbb{H}^{n}\right)} \cdot \frac{\left|B\left(u_{0}, 2^{k+1} r\right)\right|^{k / p}}{\left|B\left(u_{0}, 2^{k+1} r\right)\right|^{1 / p}}\left[1+\frac{2^{k+1} r}{\rho\left(u_{0}\right)}\right]^{\theta} .
\end{aligned}
$$

Substituting the above inequality into formula (68), we conclude that

$$
\begin{aligned}
I_{2} \leq & C\|f\|_{L_{\rho, \theta}^{p, k}\left(\mathbb{H}^{n}\right)} \sum_{k=1}^{\infty} \frac{\left|B\left(u_{0}, r\right)\right|^{(1-\kappa) / p}}{\left|B\left(u_{0}, 2^{k+1} r\right)\right|^{(1-\kappa) / p}} \\
& \cdot\left[1+\frac{2^{k+1} r}{\rho\left(u_{0}\right)}\right]^{-N \cdot\left(1 /\left(N_{0}+1\right)\right)+\theta} .
\end{aligned}
$$


By choosing some sufficiently large number $N$ such that $N>\left(N_{0}+1\right) \theta$, then we have

$$
\begin{aligned}
I_{2} & \leq C\|f\|_{L_{\rho, \theta}^{p, \kappa}\left(\mathbb{H}^{n}\right)} \sum_{k=1}^{\infty}\left(\frac{\left|B\left(u_{0}, r\right)\right|}{\left|B\left(u_{0}, 2^{k+1} r\right)\right|}\right)^{(1-\kappa) / p} \\
& \leq C\|f\|_{L_{\rho, \theta}^{p, \kappa}\left(\mathbb{H}^{n}\right)} \\
& \leq C\|f\|_{L_{\rho, \theta}^{p, \kappa}\left(\mathbb{H}^{n}\right)}\left[1+\frac{r}{\rho\left(u_{0}\right)}\right]^{\theta}
\end{aligned}
$$

where the last inequality follows from the fact that $1-$ $\kappa>0$ and $\theta>0$. Combining the above estimates for $I_{1}$ and $I_{2}$, we obtain the desired inequality (53). This ends Proof of Theorem 18.

Proof of Theorem 19. Let $f \in L_{\rho, \theta}^{1, \kappa}\left(\mathbb{H}^{n}\right)$ with $\kappa \in(0,1)$ and $\theta$ $\in(0, \infty)$. Fix $\left(u_{0}, r\right) \in \mathbb{H}^{n} \times(0, \infty)$. Our aim is to prove, by definition, that for each given ball $B=B\left(u_{0}, r\right)$ of $\mathbb{H}^{n}$, the following estimate

$\frac{1}{\left|B\left(u_{0}, r\right)\right|^{\kappa}} \sup _{\lambda>0} \lambda \cdot\left|\left\{u \in B\left(u_{0}, r\right)\left|\mathscr{T}_{\mathscr{L}}^{*}(f)(u)\right|>\lambda\right\}\right| \lesssim\left[1+\frac{r}{\rho\left(u_{0}\right)}\right]^{\theta}$,

holds true. To this end, we decompose the function $f$ as

$$
\left\{\begin{array}{l}
f=f_{1}+f_{2} \in L_{\rho, \theta}^{1, \kappa}\left(\mathbb{H}^{n}\right), \\
f_{1}=f \cdot \chi_{2 B}, \\
f_{2}=f \cdot \chi_{(2 B)^{c}} .
\end{array}\right.
$$

Then, for any fixed $\lambda>0$, we can write

$$
\begin{aligned}
& \frac{1}{\left|B\left(u_{0}, r\right)\right|^{\kappa}} \lambda \cdot\left|\left\{u \in B\left(u_{0}, r\right):\left|\mathscr{T}_{\mathscr{L}}^{*}(f)(u)\right|>\lambda\right\}\right| \\
& \leq \frac{1}{\left|B\left(u_{0}, r\right)\right|^{\kappa}} \lambda \cdot\left|\left\{u \in B\left(u_{0}, r\right):\left|\mathscr{T}_{\mathscr{L}}^{*}\left(f_{1}\right)(u)\right|>\frac{\lambda}{2}\right\}\right| \\
& \quad+\frac{1}{\left|B\left(u_{0}, r\right)\right|^{\kappa}} \lambda \cdot\left|\left\{u \in B\left(u_{0}, r\right):\left|\mathscr{T}_{\mathscr{L}}^{*}\left(f_{2}\right)(u)\right|>\frac{\lambda}{2}\right\}\right| \\
& :=J_{1}+J_{2} .
\end{aligned}
$$

We first give the estimate for the term $J_{1}$. By the second part of Theorem 9, we get

$$
\begin{aligned}
J_{1} & =\frac{1}{\left|B\left(u_{0}, r\right)\right|^{\kappa}} \lambda \cdot\left|\left\{u \in B\left(u_{0}, r\right):\left|\mathscr{T}_{\mathscr{L}}^{*}\left(f_{1}\right)(u)\right|>\frac{\lambda}{2}\right\}\right| \\
& \leq C \cdot \frac{1}{|B|^{\kappa}}\left(\int_{\mathbb{H}^{n}}\left|f_{1}(u)\right| d u\right) \\
& =C \cdot \frac{1}{|B|^{\kappa}}\left(\int_{2 B}|f(u)| d u\right) \\
& \leq C\|f\|_{L_{\rho, \theta}^{1, \kappa}\left(\mathbb{H}^{n}\right)} \cdot \frac{|2 B|^{\kappa}}{|B|^{\kappa}} \cdot\left[1+\frac{2 r}{\rho\left(u_{0}\right)}\right]^{\theta} .
\end{aligned}
$$

Therefore, in view of (57) and (10), we have

$$
J_{1} \leq C_{\theta, n}\|f\|_{L_{\rho, \theta}^{1, \kappa}\left(\mathbb{H}^{n}\right)}\left[1+\frac{r}{\rho\left(u_{0}\right)}\right]^{\theta}
$$

To estimate the second term $J_{2}$, by using the pointwise inequality (68) and Chebyshev's inequality, we can deduce that

$$
\begin{aligned}
J_{2} \leq & \frac{2}{\left|B\left(u_{0}, r\right)\right|^{\kappa}}\left(\int_{B\left(u_{0}, r\right)}\left|\mathscr{T}_{\mathscr{L}}^{*}\left(f_{2}\right)(u)\right| d u\right) \\
\leq & C \cdot \frac{\left|B\left(u_{0}, r\right)\right|}{\left|B\left(u_{0}, r\right)\right|^{\kappa}} \int_{(2 B)^{\complement}}\left[1+\frac{\left|v^{-1} u\right|}{\rho(u)}\right]^{-N} \frac{1}{\left|v^{-1} u\right|^{Q}} \cdot|f(v)| d v \\
\leq & C \cdot \frac{\left|B\left(u_{0}, r\right)\right|}{\left|B\left(u_{0}, r\right)\right|^{\kappa}} \sum_{k=1}^{\infty}\left[1+\frac{2^{k+1} r}{\rho\left(u_{0}\right)}\right]^{-N \cdot\left(1 /\left(N_{0}+1\right)\right)} \\
& \cdot \frac{1}{\left|B\left(u_{0}, 2^{k+1} r\right)\right|} \int_{B\left(u_{0}, 2^{k+1} r\right)}^{|f(v)| d v .}
\end{aligned}
$$

Moreover, for each fixed $k \in \mathbb{N}$, we compute

$$
\begin{aligned}
& \frac{1}{\left|B\left(u_{0}, 2^{k+1} r\right)\right|} \int_{B\left(u_{0}, 2^{k+1} r\right)}|f(v)| d v \\
& \leq C\|f\|_{L_{\rho, \theta}^{1, \kappa}\left(\mathbb{H}^{n}\right)} \cdot \frac{\left|B\left(u_{0}, 2^{k+1} r\right)\right|^{\kappa}}{\left|B\left(u_{0}, 2^{k+1} r\right)\right|}\left[1+\frac{2^{k+1} r}{\rho\left(u_{0}\right)}\right]^{\theta} .
\end{aligned}
$$

Consequently, substituting this inequality into formula (77),

$J_{2} \leq C\|f\|_{L_{\rho, \theta}^{1, \kappa}\left(\mathbb{H}^{n}\right)} \sum_{k=1}^{\infty} \frac{\left|B\left(u_{0}, r\right)\right|^{1-\kappa}}{\left|B\left(u_{0}, 2^{k+1} r\right)\right|^{1-\kappa}}\left[1+\frac{2^{k+1} r}{\rho\left(u_{0}\right)}\right]^{-N \cdot\left(1 /\left(N_{0}+1\right)\right)+\theta}$.

By selecting some large enough $N$ such that $N>\left(N_{0}+1\right) \theta$, we thus have

$$
\begin{aligned}
J_{2} & \leq C\|f\|_{L_{\rho, \theta}^{1, \kappa}\left(\mathbb{H}^{n}\right)} \sum_{k=1}^{\infty}\left(\frac{\left|B\left(u_{0}, r\right)\right|}{\left|B\left(u_{0}, 2^{k+1} r\right)\right|}\right)^{(1-\kappa)} \\
& \leq C\|f\|_{L_{\rho, \theta}^{1, \kappa}\left(\mathbb{H}^{n}\right)} \\
& \leq C\|f\|_{L_{\rho, \theta}^{1, \kappa}\left(\mathbb{H}^{n}\right)}\left[1+\frac{r}{\rho\left(u_{0}\right)}\right]^{\theta}
\end{aligned}
$$

where the last step is again due to the fact that $0<\kappa<1$ and $\theta$ $>0$. Summing up the above estimates for $J_{1}$ and $J_{2}$, and then taking the supremum over all $\lambda>0$, we obtain our desired result (72). This completes Proof of Theorem 19.

We also consider the maximal function with respect to the Poisson semigroup $\left\{e^{-t \sqrt{\mathscr{L}}}\right\}_{t>0}$, which is defined by

$$
\mathscr{T}_{\sqrt{\mathscr{L}}}^{*}(f)(u):=\sup _{t>0}\left|e^{-t \sqrt{\mathscr{L}}} f(u)\right|, \quad u \in \mathbb{H}^{n} .
$$


We now begin to prove that under the conditions of Theorems 18 and 19, the same results also hold for the operator $\mathscr{T}_{\sqrt{\mathscr{L}}}^{*}$ related to $\mathscr{L}$. Recall the subordination formula (see [41], p. 6)

$$
e^{-\beta}=\frac{1}{\sqrt{\pi}} \int_{0}^{\infty} \frac{e^{-v}}{\sqrt{v}} \cdot e^{-\beta^{2} / 4 v} d v
$$

Thus, from the heat semigroup, we can define the Poisson semigroup $\left\{e^{-t \sqrt{\mathscr{L}}}\right\}_{t>0}$ by

$$
e^{-t \sqrt{\mathscr{L}}} f(u)=\frac{1}{\sqrt{\pi}} \int_{0}^{\infty} \frac{e^{-v}}{\sqrt{v}} \cdot e^{-\left(t^{2} / 4 v\right) \mathscr{L}} f(u) d v
$$

From this, it follows immediately that for all $u \in \mathbb{H}^{n}$,

$$
\begin{aligned}
\mathscr{T}_{\sqrt{\mathscr{L}}}^{*}(f)(u) & \leq \sup _{t>0} \frac{1}{\sqrt{\pi}} \int_{0}^{\infty} \frac{e^{-v}}{\sqrt{v}} \cdot\left|e^{-\left(t^{2} / 4 v\right) \mathscr{L}} f(u)\right| d v \\
& \leq \frac{1}{\sqrt{\pi}} \int_{0}^{\infty} \frac{e^{-v}}{\sqrt{v}} \cdot \sup _{s>0}\left|e^{-s \mathscr{L}} f(u)\right| d v \\
& =\mathscr{T}_{\mathscr{L}}^{*}(f)(u) \cdot \frac{1}{\sqrt{\pi}} \int_{0}^{\infty} \frac{e^{-v}}{\sqrt{v}} d v=\mathscr{T}_{\mathscr{L}}^{*}(f)(u) .
\end{aligned}
$$

Hence, as an immediate consequence of Theorems 18 and 19 and Corollaries 20 and 21, we have the following results. Let $\rho$ be the same as in (14).

Theorem 28. Let $1<p<\infty, 0<\kappa<1$, and $0<\theta<\infty$. If $V$ $\in \mathscr{B}_{q}$ with $q \in[Q / 2, \infty)$, then the operator $\mathscr{T}_{\sqrt{\mathscr{L}}}^{*}$ is bounded on $L_{\rho, \theta}^{p, \kappa}\left(\mathbb{H}^{n}\right)$ and hence bounded on $L_{\rho, \infty}^{p, \kappa}\left(\mathbb{H}^{n}\right)$.

Theorem 29. Let $p=1,0<\kappa<1$, and $0<\theta<\infty$. If $V \in \mathscr{B}_{q}$ with $q \in[Q / 2, \infty)$, then the operator $\mathscr{T}_{\sqrt{\mathscr{L}}}^{*}$ is bounded from $L_{\rho, \theta}^{1, \kappa}\left(\mathbb{H}^{n}\right)$ into $W L_{\rho, \theta}^{1, \kappa}\left(\mathbb{H}^{n}\right)$ and hence bounded from $L_{\rho, \infty}^{1, \kappa}\left(\mathbb{H}^{n}\right)$ into $W L_{\rho, \infty}^{1, \kappa}\left(\mathbb{H}^{n}\right)$.

Remark 30.

(i) A slightly more general point of view is as follows. Motivated by the work in $[9,25]$, we introduce the semigroup nontangential maximal function related to $\mathscr{L}$, which is given as follows:

$$
\mathscr{T}_{\mathscr{L}}^{* *}(f)(u):=\sup _{t>0} \sup _{v \in B(u, t)}\left|e^{-t \mathscr{L}} f(v)\right|, \quad u \in \mathbb{H}^{n}
$$

It is interesting to investigate the boundedness of the operator $\mathscr{T}_{\mathscr{L}}^{* *}$ related to $\mathscr{L}$. Following the same arguments as in Proofs of Theorems 4 and 6 in [3], we are able to prove that the operator $\mathscr{T}_{\mathscr{L}}^{* *}$ is bounded on $L^{p}\left(\mathbb{H}^{n}\right)$ for all $1<p<$ $\infty$ and bounded from $L^{1}\left(\mathbb{H}^{n}\right)$ into $W L^{1}\left(\mathbb{H}^{n}\right)$. Based on this result, we can further prove that the corresponding estimates for the operator $\mathscr{T}_{\mathscr{L}}^{* *}$ remain valid in the context of Morrey spaces. The proof needs appropriate but minor modifications, and we leave this to the interested reader.

(ii) In view of the above results, we consider here the nontangential maximal function with respect to the Poisson semigroup $\left\{e^{-t \sqrt{\mathscr{L}}}\right\}_{t>0}$, which is defined by

$$
\mathscr{T}_{\sqrt{\mathscr{L}}}^{* *}(f)(u):=\sup _{t>0} \sup _{v \in B(u, t)}\left|e^{-t \sqrt{\mathscr{L}}} f(v)\right|, \quad u \in \mathbb{H}^{n} .
$$

For the same reason as above, it can be shown that this new maximal operator $\mathscr{T}_{\sqrt{\mathscr{L}}}^{* *}$ is dominated by $\mathscr{T}_{\mathscr{L}}^{* *}$ in some sense. Therefore, all the results mentioned above hold as well for the maximal operator in this more general situation.

\section{Boundedness of the Riesz Transforms}

This section is concerned with Proofs of Theorems 22, 23, and 24 . Recall that the operators $\mathscr{R}_{\mathscr{L}}$ and $\mathscr{R}_{\mathscr{L}}^{*}$ have singular kernels with values in $\mathbb{R}^{2 n}$ that will be denoted by $\mathscr{K}(u, v)$ and $\mathscr{K}^{*}(u, v)$, respectively (see [3]).

$$
\left\{\begin{array}{l}
\mathscr{R}_{\mathscr{L}}(f)(u)=\nabla_{\mathbb{H}^{n}} \mathscr{L}^{-1 / 2}(f)(u)=\int_{\mathbb{H}^{n}} \mathscr{K}(u, v) f(v) d v, \\
\mathscr{R}_{\mathscr{L}}^{*}(f)(u)=\mathscr{L}^{-1 / 2} \nabla_{\mathbb{H}^{n}}(f)(u)=\int_{\mathbb{H}^{n}} \mathscr{K}^{*}(u, v) f(v) d v .
\end{array}\right.
$$

Obviously,

$$
\begin{gathered}
\mathscr{K}^{*}(u, v)=-\mathscr{K}(v, u) \\
\left|\mathscr{K}^{*}(u, v)\right|=|\mathscr{K}(v, u)| .
\end{gathered}
$$

The next lemma plays a crucial role in our Proofs of Theorems 22-24.

Lemma 31. Let $V \in \mathscr{B}_{q}$ with $q \in[Q / 2, Q)$, and let $\rho(\cdot)$ be the auxiliary function determined by $V$. For every positive integer $N \in \mathbb{N}$, there exists a positive constant $C_{N}>0$ such that, for any $u$ and $v$ in $\mathbb{H}^{n}$,

$$
\begin{aligned}
\left|\mathscr{K}^{*}(u, v)\right| \leq & C_{N}\left[1+\frac{\left|v^{-1} u\right|}{\rho(u)}\right]^{-N} \frac{1}{\| v^{-1} u^{Q-1}} \\
& \times\left\{\int_{B\left(v,\left|v^{-1} u\right| / 4\right)} \frac{V(w)}{\left|v^{-1} w\right|^{Q-1}} d w+\frac{1}{\left|v^{-1} u\right|}\right\},
\end{aligned}
$$

where $\mathscr{K}^{*}(u, v)$ denotes the (vector-valued) kernel of the operator $\mathscr{R}_{\mathscr{L}}^{*}$. Moreover, the above inequality also holds with $\rho(u)$ replaced by $\rho(v)$.

Lemma 31 was proved by $\mathrm{Li}$ [21] in a more general setting (connected nilpotent Lie group) (see also [19] for $\mathbb{H}^{n}$ ). For 
such kernels, we also give the following result, which establishes the Lipschitz regularity of $\mathscr{K}^{*}(u, v)$.

Lemma 32. Let $V \in \mathscr{B}_{q}$ with $q \in[Q / 2, Q)$, and let $\rho(\cdot)$ be the auxiliary function determined by $V$. For every positive integer $N \in \mathbb{N}$, there exists a positive constant $C_{N}>0$ such that, for any $u$ and $v$ in $\mathbb{H}^{n}$, and for some fixed $\delta^{\prime} \in(0, \delta), \delta$ is given as in (16),

$$
\begin{aligned}
& \left|\mathscr{K}^{*}(u h, v)-\mathscr{K}^{*}(u, v)\right| \\
& \leq C_{N}\left[1+\frac{\left|v^{-1} u\right|}{\rho(u)}\right]^{-N} \frac{|h|^{\delta^{\prime}}}{\left|v^{-1} u\right|^{Q-1+\delta^{\prime}}} \\
& \quad \times\left\{\int_{B\left(v,\left|v^{-1} u\right| / 4\right)} \frac{V(w)}{\left|v^{-1} w\right|^{Q-1}} d w+\frac{1}{\left|v^{-1} u\right|}\right\},
\end{aligned}
$$

whenever $|h| \leq\left|v^{-1} u\right| / 4 /$. Moreover, the above inequality also holds with $\rho(u)$ replaced by $\rho(v)$.

The above kernel estimate in Lemma 32 was obtained by Pengtao and Lizhong in [19], which will be used to prove that the operator $\mathscr{R}_{\mathscr{L}}$ is of weak-type $(1,1)$. Recall that in the Euclidean setting, the kernel estimates (89) and (90) were proved in $[10,42]$.

We are now in a position to give the proofs of our main theorems.

Proof of Theorem 22. Let $f \in L_{\rho, \theta}^{p, \kappa}\left(\mathbb{H}^{n}\right)$ with $(p, \kappa) \in\left(p^{\prime}{ }_{0}, \infty\right)$ $\times(0,1)$ and $\theta \in(0, \infty)$, where $1 / p_{0}=1 / q-1 / Q$. Fix $\left(u_{0}, r\right)$ $\in \mathbb{H}^{n} \times(0, \infty)$. By definition, we only need to show that for any given ball $B=B\left(u_{0}, r\right)$ of $\mathbb{H}^{n}$, the following inequality

$$
\left(\frac{1}{\left|B\left(u_{0}, r\right)\right|^{\kappa}} \int_{B\left(u_{0}, r\right)}\left|\mathscr{R}_{\mathscr{L}}^{*}(f)(u)\right|^{p} d u\right)^{1 / p} \lesssim\left[1+\frac{r}{\rho\left(u_{0}\right)}\right]^{\theta},
$$

holds true. Using the standard technique, we decompose the function $f$ as

$$
\left\{\begin{array}{l}
f=f_{1}+f_{2} \in L_{\rho, \theta}^{p, \kappa}\left(\mathbb{H}^{n}\right), \\
f_{1}=f \cdot \chi_{2 B}, \\
f_{2}=f \cdot \chi_{(2 B)^{\complement}} \cdot
\end{array}\right.
$$

Then, by using the linearity of $\mathscr{R}_{\mathscr{L}}^{*}$, we write

$$
\begin{aligned}
& \left(\frac{1}{\left|B\left(u_{0}, r\right)\right|^{\kappa}} \int_{B\left(u_{0}, r\right)}\left|\mathscr{R}_{\mathscr{L}}^{*}(f)(u)\right|^{p} d u\right)^{1 / p} \\
& \leq\left(\frac{1}{\left|B\left(u_{0}, r\right)\right|^{\kappa}} \int_{B\left(u_{0}, r\right)}\left|\mathscr{R}_{\mathscr{L}}^{*}\left(f_{1}\right)(u)\right|^{p} d u\right)^{1 / p} \\
& \quad+\left(\frac{1}{\left|B\left(u_{0}, r\right)\right|^{\kappa}} \int_{B\left(u_{0}, r\right)}\left|\mathscr{R}_{\mathscr{L}}^{*}\left(f_{2}\right)(u)\right|^{p} d u\right)^{1 / p}:=K_{1}+K_{2} .
\end{aligned}
$$

Let us consider the first term $K_{1}$. Making use of (10), (57), and Theorem 11, we have

$$
\begin{aligned}
K_{1} & \leq C \cdot \frac{1}{|B|^{\kappa / p}}\left(\int_{\mathbb{H}^{n}}\left|f_{1}(u)\right|^{p} d u\right)^{1 / p} \\
& =C \cdot \frac{1}{|B|^{\kappa / p}}\left(\int_{2 B}|f(u)|^{p} d u\right)^{1 / p} \\
& \leq C\|f\|_{L_{\rho, \theta}^{p, \kappa}\left(\mathbb{H}^{n}\right)} \cdot \frac{|2 B|^{\kappa / p}}{|B|^{\kappa / p}} \cdot\left[1+\frac{2 r}{\rho\left(u_{0}\right)}\right]^{\theta} \\
& \leq C_{\theta, n}\|f\|_{L_{\rho, \theta}^{p, \kappa}\left(\mathbb{H}^{n}\right)}\left[1+\frac{r}{\rho\left(u_{0}\right)}\right]^{\theta} .
\end{aligned}
$$

Now let us turn to estimate the second term $K_{2}$. By using Lemma 31 , we obtain that for any $u \in B\left(u_{0}, r\right)$,

$$
\left|\mathscr{R}_{\mathscr{L}}^{*}\left(f_{2}\right)(u)\right|=\left|\int_{(2 B)^{\complement}} \mathscr{K}^{*}(u, v) f(v) d v\right| \leq C_{N}[\mathbf{I}(u)+\mathbf{I I}(u)],
$$

where

$$
\begin{aligned}
\mathbf{I}(u):= & \int_{(2 B)^{\complement}}\left[1+\frac{\left|v^{-1} u\right|}{\rho(u)}\right]^{-N} \frac{1}{\left|v^{-1} u\right|^{Q}} \cdot|f(v)| d v, \\
\mathbf{I I}(u):= & \int_{(2 B)^{\complement}}\left[1+\frac{\left|v^{-1} u\right|}{\rho(u)}\right]^{-N} \frac{1}{\| v^{-1} u^{Q-1}} \\
& \cdot\left\{\int_{B\left(v,\left|v^{-1} u\right| / 4\right)} \frac{V(w)}{\left|v^{-1} w\right|^{Q-1}} d w\right\} \cdot|f(v)| d v .
\end{aligned}
$$

Hence, $K_{2}$ can be written as follows:

$$
\begin{aligned}
K_{2}= & \left(\frac{1}{\left|B\left(u_{0}, r\right)\right|^{\kappa}} \int_{B\left(u_{0}, r\right)}\left|\mathscr{R}_{\mathscr{L}}^{*}\left(f_{2}\right)(u)\right|^{p} d u\right)^{1 / p} \\
\lesssim & \left(\frac{1}{\left|B\left(u_{0}, r\right)\right|^{\kappa}} \int_{B\left(u_{0}, r\right)}|\mathbf{I}(u)|^{p} d u\right)^{1 / p} \\
& +\left(\frac{1}{\left|B\left(u_{0}, r\right)\right|^{\kappa}} \int_{B\left(u_{0}, r\right)}|\mathbf{I I}(u)|^{p} d u\right)^{1 / p}:=K_{3}+K_{4} .
\end{aligned}
$$

Arguing as in Proof of Theorem 18, we can also obtain

$$
K_{3} \leq C\|f\|_{L_{\rho, \theta}^{p, \kappa}\left(\mathbb{H}^{n}\right)}\left[1+\frac{r}{\rho\left(u_{0}\right)}\right]^{\theta}
$$

We only have to deal with the term $K_{4}$. As mentioned in the previous proof, one has

$$
\frac{1}{2}\left|v^{-1} u_{0}\right| \leq\left|v^{-1} u\right| \leq \frac{3}{2}\left|v^{-1} u_{0}\right|,
$$


whenever $u \in B\left(u_{0}, r\right)$ and $v \in(2 B)^{\complement}$. Thus, for any positive integer $N \in \mathbb{N}$,

$$
\begin{aligned}
& |\mathbf{I I}(u)| \lesssim \int_{(2 B)^{\complement}}\left[1+\frac{\left|v^{-1} u_{0}\right|}{\rho(u)}\right]^{-N} \frac{1}{\left|v^{-1} u_{0}\right|^{Q-1}} \\
& \cdot\left|\int_{B\left(v,\left|v^{-1} u\right| / 4\right)} \frac{V(w)}{\left|v^{-1} w\right|^{Q-1}} d w\right| \cdot|f(v)| d v \\
& =\sum_{k=1}^{\infty} \int_{2^{k} r \leq\left|v^{-1} u_{0}\right|<2^{k+1} r}\left[1+\frac{\left|v^{-1} u_{0}\right|}{\rho(u)}\right]^{-N} \frac{1}{\left|v^{-1} u_{0}\right|^{Q-1}} \\
& \times\left|\int_{B\left(v,\left|v^{-1} u\right| / 4\right)} \frac{V(w)}{\left|v^{-1} w\right|^{Q-1}} d \mathrm{w}\right| \cdot|f(v)| d v \\
& \lesssim \sum_{k=1}^{\infty} \frac{1}{\left|B\left(u_{0}, 2^{k+1} r\right)\right|^{1-1 / Q}} \int_{B\left(u_{0}, 2^{k+1} r\right)}\left[1+\frac{2^{k} r}{\rho(u)}\right]^{-N} \\
& \times\left|\int_{B\left(v,\left|v^{-1} u\right| / 4\right)} \frac{V(w)}{\left|v^{-1} w\right|^{Q-1}} d w\right| \cdot|f(v)| d v .
\end{aligned}
$$

It is not difficult to check that when $w \in B\left(v,\left|v^{-1} u\right| / 4\right)$ and $v \in B\left(u_{0}, 2^{k+1} r\right)$, one has $w \in B\left(u_{0}, 2^{k+2} r\right)$, which implies

$$
\begin{aligned}
|\mathbf{I I}(u)| \lesssim & \sum_{k=1}^{\infty} \frac{1}{\left|B\left(u_{0}, 2^{k+1} r\right)\right|^{1-1 / Q}}\left[1+\frac{2^{k} r}{\rho(u)}\right]^{-N} \\
& \times \int_{B\left(u_{0}, 2^{k+1} r\right)}\left|\mathscr{I}_{1}\left(V \chi_{B\left(u_{0}, 2^{k+2} r\right)}\right)(v)\right| \cdot|f(v)| d v
\end{aligned}
$$

where $\mathscr{I}_{1}$ stands for the fractional integral operator of order one defined by

$$
\mathscr{I}_{1} g(v):=\int_{\mathbb{H}^{n}} \frac{g(w)}{\left|v^{-1} w\right|^{Q-1}} d w
$$

For this operator, a classical result of Folland and Stein [43] states that $\mathscr{I}_{1}$ is bounded from $L^{q}\left(\mathbb{H}^{n}\right)$ into $L^{p_{0}}\left(\mathbb{H}^{n}\right)$ for $1<q<Q$ and $1 / p_{0}=1 / q-1 / Q$ (see also [39, 44]). Namely, there exists a constant $C>0$ such that for any $g \in L^{q}\left(\mathbb{H}^{n}\right)$,

$$
\left\|\mathscr{I}_{1}(g)\right\|_{L^{p_{0}\left(\mathbb{H}^{n}\right)}} \leq C\|g\|_{L^{q}\left(\mathbb{H}^{n}\right)} .
$$

Since $\left(p_{0}\right)^{\prime}<p<\infty$, we can choose a number $s>0$ such that $1 / p+1 / p_{0}+1 / s=1$. A combination of the
Hölder inequality and (103) gives

$$
\begin{aligned}
& \int_{B\left(u_{0}, 2^{k+1} r\right)}\left|\mathscr{I}_{1}\left(V \chi_{B\left(u_{0}, 2^{k+2} r\right)}\right)(v)\right| \cdot|f(v)| d v \\
& \leq\left(\int_{B\left(u_{0}, 2^{k+1} r\right)}|f(v)|^{p} d v\right)^{1 / p}\left(\int_{B\left(u_{0}, 2^{k+1} r\right)} 1 d v\right)^{1 / s} \\
& \times\left(\int_{B\left(u_{0}, 2^{k+1} r\right)}\left|\mathscr{I}_{1}\left(V \chi_{B\left(u_{0}, 2^{k+2} r\right)}\right)(v)\right|^{p_{0}} d v\right)^{1 / p_{0}} \\
& \leq C\left(\int_{B\left(u_{0}, 2^{k+1} r\right)}|f(v)|^{p} d v\right)^{1 / p}\left|B\left(u_{0}, 2^{k+1} r\right)\right|^{1 / s} \\
& \times\left(\int_{B\left(u_{0}, 2^{k+2} r\right)} V(w)^{q} d w\right)^{1 / q} \\
& \leq C\left(\int_{B\left(u_{0}, 2^{k+1} r\right)}|f(v)|^{p} d v\right)^{1 / p}\left|B\left(u_{0}, 2^{k+1} r\right)\right|^{1 / s} \\
& \times\left(\frac{1}{\left|B\left(u_{0}, 2^{k+2} r\right)\right|^{1 / q^{\prime}}} \int_{B\left(u_{0}, 2^{k+2} r\right)} V(w) d w\right),
\end{aligned}
$$

where the last inequality holds by our assumption $V \in \mathscr{B}_{q}$. We now claim that the following inequality holds. For any $N_{1}>\log _{2} C_{0}\left(C_{0}\right.$ is the doubling constant in Lemma 3$)$, there exists a constant $C>0$ such that for any $u_{0} \in \mathbb{H}^{n}$ and $\tau \in(0, \infty)$,

$$
\int_{B\left(u_{0}, \tau\right)} V(w) d w \leq C \cdot \tau^{Q-2}\left[1+\frac{\tau}{\rho\left(u_{0}\right)}\right]^{N_{1}}
$$

Taking this claim momentarily for granted, then we have

$$
\begin{aligned}
& \int_{B\left(u_{0}, 2^{k+1} r\right)}\left|\mathscr{I}_{1}\left(V \chi_{B\left(u_{0}, 2^{k+2} r\right)}\right)(v)\right| \cdot|f(v)| d v \\
& \leq C\left(\int_{B\left(u_{0}, 2^{k+1} r\right)}|f(v)|^{p} d v\right)^{1 / p}\left|B\left(u_{0}, 2^{k+1} r\right)\right|^{1 / s} \\
& \cdot \frac{\left(2^{k+2} r\right)}{\left|B\left(u_{0}, 2^{k+2} r\right)\right|^{1 / q^{\prime}}}\left[1+\frac{2^{k+2} r}{\rho\left(u_{0}\right)}\right]^{N_{1}} \\
& \leq C\left(\int_{B\left(u_{0}, 2^{k+1} r\right)}|f(v)|^{p} d v\right)^{1 / p} \cdot \frac{1}{\left|B\left(u_{0}, 2^{k+1} r\right)\right|^{1 / q^{\prime}-1 / s-1+2 / Q}} \\
& \times {\left[1+\frac{2^{k+1} r}{\rho\left(u_{0}\right)}\right]^{N_{1}}, }
\end{aligned}
$$


where in the last step we have invoked (57) and (10). In addition, it follows immediately from (21) and (57) that

$$
\begin{aligned}
{\left[1+\frac{2^{k} r}{\rho(u)}\right]^{-N} } & \leq\left[1+\frac{r}{\rho\left(u_{0}\right)}\right]^{N \cdot\left(N_{0} /\left(N_{0}+1\right)\right)}\left[1+\frac{2^{k} r}{\rho\left(u_{0}\right)}\right]^{-N} \\
& \leq\left[1+\frac{2^{k} r}{\rho\left(u_{0}\right)}\right]^{-N \cdot\left(1 /\left(N_{0}+1\right)\right)} \\
& \leq\left[1+\frac{2^{k+1} r}{\rho\left(u_{0}\right)}\right]^{-N \cdot\left(1 /\left(N_{0}+1\right)\right)} .
\end{aligned}
$$

A trivial computation shows that

$$
\left(1-\frac{1}{Q}\right)+\left(\frac{1}{q^{\prime}}-\frac{1}{s}-1+\frac{2}{Q}\right)=\frac{1}{Q}+\frac{1}{q^{\prime}}-\frac{1}{s}=\frac{1}{p} .
$$
that

Therefore, in view of (108) and (107), we conclude

$$
\begin{aligned}
|\mathbf{I I}(u)| \lesssim & \sum_{k=1}^{\infty} \frac{1}{\left|B\left(u_{0}, 2^{k+1} r\right)\right|^{1 / p}}\left[1+\frac{2^{k+1} r}{\rho\left(u_{0}\right)}\right]^{-N \cdot\left(1 /\left(N_{0}+1\right)\right)+N_{1}} \\
& \times\left(\int_{B\left(u_{0}, 2^{k+1} r\right)}|f(v)|^{p} d v\right)^{1 / p} \\
\lesssim & \|f\|_{L_{\rho, \theta}^{p, k}\left(\mathbb{H}^{n}\right)} \sum_{k=1}^{\infty} \frac{\left|B\left(u_{0}, 2^{k+1} r\right)\right|^{\kappa / p}}{\left|B\left(u_{0}, 2^{k+1} r\right)\right|^{1 / p}} \\
& \cdot\left[1+\frac{2^{k+1} r}{\rho\left(u_{0}\right)}\right]^{-N \cdot\left(1 /\left(N_{0}+1\right)\right)+N_{1}+\theta}
\end{aligned}
$$

Consequently,

$$
\begin{aligned}
K_{4} \leq & C\|f\|_{L_{\rho, \theta}^{p, k}\left(\mathbb{H}^{n}\right)} \sum_{k=1}^{\infty} \frac{\left|B\left(u_{0}, r\right)\right|^{(1-\kappa) / p}}{\left|B\left(u_{0}, 2^{k+1} r\right)\right|^{(1-\kappa) / p}} \\
\cdot\left[1+\frac{2^{k+1} r}{\rho\left(u_{0}\right)}\right]^{-N \cdot\left(\left(1 /\left(N_{0}+1\right)\right)+N_{1}+\theta\right.} & .
\end{aligned}
$$

By choosing some sufficiently large number $N$ such that $N>\left(N_{0}+1\right)\left(N_{1}+\theta\right)$, then we have

$$
\begin{aligned}
K_{4} & \leq C\|f\|_{L_{\rho, \theta}^{p, \kappa}\left(\mathbb{H}^{n}\right)} \sum_{k=1}^{\infty}\left(\frac{\left|B\left(u_{0}, r\right)\right|}{\left|B\left(u_{0}, 2^{k+1} r\right)\right|}\right)^{(1-\kappa) / p} \\
& \leq C\|f\|_{L_{\rho, \theta}^{p, \kappa}\left(\mathbb{H}^{n}\right)} \leq C\|f\|_{L_{\rho, \theta}^{p, \kappa}\left(\mathbb{H}^{n}\right)}\left[1+\frac{r}{\rho\left(u_{0}\right)}\right]^{\theta},
\end{aligned}
$$

where the last inequality follows again from the fact that $1-\kappa>0$ and $\theta>0$. Combining the above estimates for $K_{1}, K_{3}$, and $K_{4}$ produces the desired inequality (91).
Finally, let us verify (105). Suppose that $V \in \mathscr{B}_{q}$ with $Q /$ $2 \leq q<Q$. Using the same method as in Proof of Lemma 1 in [42], for any $u_{0} \in \mathbb{H}^{n}$ and $\tau \in(0, \infty)$, there must exist an integer $j$ such that $2^{j} \tau \leq \rho\left(u_{0}\right)<2^{j+1} \tau$. Two cases are considered below.

Case 1. $j<0$. In this case, one has $\rho\left(u_{0}\right)<\tau$. This fact together with Lemmas 3 and 4 yields

$$
\begin{aligned}
& {\left[1+\frac{\tau}{\rho\left(u_{0}\right)}\right]^{-N_{1}} \int_{B\left(u_{0}, \tau\right)} V(w) d w} \\
& \quad \leq\left(2^{-j}\right)^{-N_{1}} \int_{B\left(u_{0}, 2^{-j} \rho\left(u_{0}\right)\right)} V(w) d w \\
& \quad \leq\left(2^{-j}\right)^{-N_{1}}\left(C_{0}\right)^{-j} \int_{B\left(u_{0}, \rho\left(u_{0}\right)\right)} V(w) d w \\
& \quad \leq\left(2^{-j}\right)^{-N_{1}}\left(C_{0}\right)^{-j} \tau^{Q-2} .
\end{aligned}
$$

Since $N_{1}>\log _{2} C_{0}$, it is easy to see that

$$
\left[1+\frac{\tau}{\rho\left(u_{0}\right)}\right]^{-N_{1}} \int_{B\left(u_{0}, \tau\right)} V(w) d w \leq \tau^{Q-2} .
$$

Case 2. $j \geq 0$. In this case, one has $\tau \leq \rho\left(u_{0}\right)$. This fact, along with Lemmas 5 and 4 , implies that

$$
\begin{aligned}
& {\left[1+\frac{\tau}{\rho\left(u_{0}\right)}\right]^{-N_{1}} \int_{B\left(u_{0}, \tau\right)} V(w) d w} \\
& \quad \leq \int_{B\left(u_{0}, \tau\right)} V(w) d w=\tau^{Q-2} \cdot \frac{1}{\tau^{Q-2}} \int_{B\left(u_{0}, \tau\right)} V(w) d w \\
& \quad \leq C \cdot \tau^{Q-2} \cdot \frac{1}{\rho\left(u_{0}\right)^{Q-2}} \int_{B\left(u_{0}, \rho\left(u_{0}\right)\right)} V(w) d w=C \cdot \tau^{Q-2} .
\end{aligned}
$$

Thus, in both cases, (105) holds. This completes Proof of Theorem 22.

In order to prove Theorem 24, let us first set up the following result, which is based on a version of the CalderónZygmund decomposition on $\mathbb{H}^{n}$ and Lemma 32.

Theorem 33. Let $V \in \mathscr{B}_{q}$ with $q \in[Q / 2, Q)$. Then, the Riesz transform $\mathscr{R}_{\mathscr{L}}$ is bounded from $L^{1}\left(\mathbb{H}^{n}\right)$ into $W L^{1}\left(\mathbb{H}^{n}\right)$.

Proof. For any given $f \in L^{1}\left(\mathbb{H}^{n}\right)$ and $\sigma>0$, making use of the Calderón-Zygmund decomposition of $f$ at height $\sigma$ (see [43]), we have the decomposition $f=g+b$ with $b=\sum_{i} b_{i}$ such that

(1) $|g(u)| \leq C \cdot \sigma$, for almost every $u \in \mathbb{H}^{n}$ and

$$
\|g\|_{L^{1}\left(\mathbb{H}^{n}\right)} \leq C\|f\|_{L^{1}\left(\mathbb{H}^{n}\right)},
$$


(2) each $b_{i}$ is supported in the ball $B_{i}=B\left(u_{i}, r_{i}\right)$, and we denote the center and the radius of $B_{i}$ by $u_{i}$ and $r_{i}$, respectively

$$
\begin{aligned}
\int_{B_{i}} b_{i}(u) d u & =0, \\
b_{i} \|_{L^{1}} & \leq C \sigma\left|B_{i}\right|,
\end{aligned}
$$

(3) the sets $B_{i}$ are finitely overlapping and

$$
\sum_{i}\left|B_{i}\right| \leq \frac{C}{\sigma}\|f\|_{L^{1}\left(\mathbb{H}^{n}\right)} .
$$

From this construction, we have that for any fixed $\sigma>0$,

$$
\begin{aligned}
& \left|\left\{u \in \mathbb{H}^{n}:\left|\mathscr{R}_{\mathscr{L}}(f)(u)\right|>\sigma\right\}\right| \\
& \leq\left|\left\{u \in \mathbb{H}^{n}:\left|\mathscr{R}_{\mathscr{L}}(g)(u)\right|>\frac{\sigma}{2}\right\}\right| \\
& \quad+\left|\left\{u \in \mathbb{H}^{n}:\left|\mathscr{R}_{\mathscr{L}}(b)(u)\right|>\sigma / 2\right\}\right|:=\mathbf{I}+\mathbf{I I} .
\end{aligned}
$$

The part of the argument involving the function $g$ proceeds as follows. By using the $L^{2}\left(\mathbb{H}^{n}\right)$ boundedness of $\mathscr{R}_{\mathscr{L}}$ (see Theorem 12 with $1<2<p_{0}$ ), we obtain

$$
\mathbf{I} \leq \frac{C}{\sigma^{2}}\|f\|_{L^{2}\left(\mathbb{H}^{n}\right)}^{2} \leq \frac{C}{\sigma}\|g\|_{L^{1}\left(\mathbb{H}^{n}\right)} \leq \frac{C}{\sigma}\|f\|_{L^{1}\left(\mathbb{H}^{n}\right)} .
$$

Setting $E=\bigcup_{i} 4 B_{i}=\bigcup_{i} B\left(u_{i}, 4 r_{i}\right)$, we split II into two parts as follows:

$$
\begin{aligned}
\mathbf{I I} \leq & \left|\left\{u \in E:\left|\mathscr{R}_{\mathscr{L}}(b)(u)\right|>\frac{\sigma}{2}\right\}\right| \\
& +\left|\left\{u \in E^{\complement}:\left|\mathscr{R}_{\mathscr{L}}(b)(u)\right|>\frac{\sigma}{2}\right\}\right|:=\mathbf{I I I}+\mathbf{I V} .
\end{aligned}
$$

It is obvious that

$$
\mathbf{I I I} \leq\left|\bigcup_{i} 4 B_{i}\right| \leq C \sum_{i}\left|B_{i}\right| \leq \frac{C}{\sigma}\|f\|_{L^{1}\left(\mathbb{H}^{n}\right)}
$$

Therefore, in order to complete our proof, we need only to show that

$$
\mathbf{I V} \leq \frac{C}{\sigma}\|f\|_{L^{1}\left(\mathbb{H}^{n}\right)}
$$

An application of Chebyshev's inequality yields

$\mathbf{I V} \leq \frac{2}{\sigma} \int_{E^{\complement}}\left|\mathscr{R}_{\mathscr{L}}(b)(u)\right| d u \leq \frac{2}{\sigma} \sum_{i} \int_{\left(4 B_{i}\right)^{\complement}}\left|\mathscr{R}_{\mathscr{L}}\left(b_{i}\right)(u)\right| d u$.

We observe that

$$
\left|u_{i}^{-1} v\right|<r_{i}<\frac{\left|u_{i}^{-1} u\right|}{4}
$$

whenever $v \in B_{i}$ and $u \in\left(4 B_{i}\right)^{\complement}$. Then, we apply Lemma 32, (88), and the cancelation condition of $b_{i}$ to get

$$
\begin{aligned}
\left|\mathscr{R}_{\mathscr{L}}\left(b_{i}\right)(u)\right|= & \left|\int_{\mathbb{H}^{n}} \mathscr{K}(u, v) b_{i}(v) d v\right| \\
= & \left|\int_{B_{i}}\left[\mathscr{K}(u, v)-\mathscr{K}\left(u, u_{i}\right)\right] b_{i}(v) d v\right| \\
\leq & \int_{B_{i}}\left|\mathscr{K}^{*}(v, u)-\mathscr{K}^{*}\left(u_{i}, u\right)\right|\left|b_{i}(v)\right| d v \\
\leq & \int_{B_{i}} \frac{\left|u_{i}^{-1} v\right|^{\delta^{\prime}}}{\left|u_{i}^{-1} u\right|^{Q+\delta^{\prime}}}\left|b_{i}(v)\right| d v+\int_{B_{i}}\left[1+\frac{\left|u_{i}^{-1} u\right|}{\rho\left(u_{i}\right)}\right]^{-N} \\
& \cdot\left\{\int_{B\left(u,\left|u_{i}^{-1} u\right| / 4\right)} \frac{V(w)}{\left|u^{-1} w\right|^{Q-1}} d w\right\} \\
& \cdot \frac{\left|u_{i}^{-1} v\right|^{\delta^{\prime}}}{\left|u_{i}^{-1} \mathrm{u}\right|^{Q-1+\delta^{\prime}}}\left|b_{i}(v)\right| d v .
\end{aligned}
$$

Obviously, the first term on the right-hand side of (125) is bounded by

$$
\int_{B_{i}} \frac{r_{i}^{\delta}}{\left|u_{i}^{-1} u\right|^{Q+\delta^{\prime}}}\left|b_{i}(v)\right| d v \leq r_{i}^{\delta}\left\|b_{i}\right\|_{L^{1}} \cdot \frac{1}{\left|u_{i}^{-1} u\right|^{Q+\delta^{\prime}}} .
$$

Using this estimate together with (11), we can deduce that

$$
\begin{aligned}
\mathbf{I V}^{(1)} & :=\frac{1}{\sigma} \sum_{i} \int_{\left(4 B_{i}\right)^{\complement}}\left\{\int_{B_{i}\left|u_{i}^{-1} u\right|^{Q+\delta^{\prime}}} \frac{\left|u_{i}^{-1} v\right|^{\delta^{\prime}}}{}(v) \mid d v\right\} d u \\
& \leq \frac{1}{\sigma} \sum_{i} r_{i}^{\delta}\left\|b_{i}\right\|_{L^{1}} \int_{\left|u_{i}^{-1} u\right|>4 r_{i}\left|u_{i}^{-1} u\right|^{Q+\delta^{\prime}}} \frac{d u}{} \\
& =\frac{1}{\sigma} \sum_{i} r_{i}^{\delta}\left|b_{i}\right|_{L^{1}} \int_{4 r_{i}}^{\infty} \frac{1}{\rho^{Q+\delta^{\prime}}} \cdot \rho^{Q-1} d \rho \\
& \leq \frac{C}{\sigma} \sum_{i} \sigma\left|B_{i}\right| \leq \frac{C}{\sigma}\|f\|_{L^{1}\left(\mathbb{H}^{n}\right)} .
\end{aligned}
$$

On the other hand, the latter term on the right-hand side of (125) is controlled by

$$
\begin{aligned}
\int_{B_{i}}[1 & \left.+\frac{\left|u_{i}^{-1} u\right|}{\rho\left(u_{i}\right)}\right]^{-N}\left\{\int_{B\left(u,\left|u_{i}^{-1} u\right| / 4\right)} \frac{V(w)}{\left|u^{-1} w\right|^{Q-1}} d w\right\} \\
& \times \frac{r_{i}^{\delta}}{\left|u_{i}^{-1} u\right|^{Q-1+\delta^{\prime}}}\left|b_{i}(v)\right| d v \\
\leq & r_{i}^{\delta}\left\|b_{i}\right\|_{L^{1}} \cdot \frac{1}{\left|u_{i}^{-1} u\right|^{Q-1+\delta^{\prime}}}\left[1+\frac{\left|u_{i}^{-1} u\right|}{\rho\left(u_{i}\right)}\right]^{-N} \\
& \times\left\{\int_{B\left(u,\left|u_{i}^{-1} u\right| / 4\right)} \frac{V(w)}{\left|u^{-1} w\right|^{Q-1}} d w\right\} .
\end{aligned}
$$


When $w \in B\left(u,\left|u_{i}^{-1} u\right| / 4\right)$, by the triangle inequality, one has

$$
\left|u_{i}^{-1} w\right| \leq\left|u_{i}^{-1} u\right|+\left|u^{-1} w\right| \leq 2\left|u_{i}^{-1} u\right| \text {. }
$$

From this, it follows that the above expression is bounded by

$r_{i}^{\delta}|| b_{i} \|_{L^{1}} \cdot \frac{1}{\left|u_{i}^{-1} u\right|^{Q-1+\delta^{\prime}}}\left[1+\frac{\left|u_{i}^{-1} u\right|}{\rho\left(u_{i}\right)}\right]^{-N}\left\{\int_{B\left(u_{i}, 2\left|u_{i}^{-1} u\right|\right)} \frac{V(w)}{\left|u^{-1} w\right|^{Q-1}} d w\right\}$.

Consequently,

$$
\begin{aligned}
\mathbf{I V}^{(2)}:= & \frac{1}{\sigma} \sum_{i} \int_{\left(4 B_{i}\right)^{\mathrm{C}}} \int_{B_{i}}\left[1+\frac{\left|u_{i}^{-1} u\right|}{\rho\left(u_{i}\right)}\right]^{-N} \\
& \times\left\{\int_{B\left(u,\left|u_{i}^{-1} u\right| / 4\right)} \frac{V(w)}{\left|u^{-1} w\right|^{Q-1}} d w\right\} \frac{\left|u_{i}^{-1} v\right|^{\delta^{\prime}}}{\left|u_{i}^{-1} u\right|^{Q-1+\delta^{\prime}}} \\
& \cdot\left|b_{i}(v)\right| d v d u \\
\leq & \frac{1}{\sigma} \sum_{i} \int_{\left(4 B_{i}\right)^{\mathrm{C}}} r_{i}^{\delta}\left\|b_{i}\right\|_{L^{1}} \cdot \frac{1}{\left|u_{i}^{-1} u\right|^{Q-1+\delta^{\prime}}}\left[1+\frac{\left|u_{i}^{-1} u\right|}{\rho\left(u_{i}\right)}\right]^{-N} \\
& \times\left\{\int_{B\left(u_{i}, 2\left|u_{i}^{-1} u\right|\right)} \frac{V(w)}{\left|u^{-1} w\right|^{Q-1}} d w\right\} d u \\
\leq & \frac{1}{\sigma} \sum_{i} r_{i}^{\delta}\left\|\mid b_{i}\right\|_{L^{1}} \sum_{k=1}^{\infty}\left[1+\frac{2^{k+1} r_{i}}{\rho\left(u_{i}\right)}\right]^{-N} \times \int_{2^{k+1} r_{i} \leq\left|u_{i}^{-1} u\right|<2^{k+2} r_{i}} \\
& \cdot \frac{1}{\left|u_{i}^{-1} u\right|^{Q-1+\delta^{\prime}}} \cdot\left|\mathscr{I}_{1}\left(V \chi_{B\left(u_{i} 2^{k+3} r_{i}\right)}\right)(u)\right| d u,
\end{aligned}
$$

where $\mathscr{I}_{1}$ is the fractional integral operator of order one given in (102). A combination of the Hölder inequality and (103) implies that for each fixed $k \in \mathbb{N}$,

$$
\begin{aligned}
& \int_{2^{k+1} r_{i} \leq\left|u_{i}^{-1} u\right|<2^{k+2} r_{i}} \frac{1}{\left|u_{i}^{-1} u\right|^{Q-1+\delta^{\prime}}} \cdot\left|\mathscr{I}_{1}\left(V \chi_{B\left(u_{i} 2^{k+3} r_{i}\right)}\right)(u)\right| d u \\
& \leq\left(\int_{2^{k+1} r_{i} \leq\left|u_{i}^{-1} u\right|<2^{k+2} r_{i}} \frac{1}{\left|u_{i}^{-1} u\right|^{\left(\mathrm{Q}-1+\delta^{\prime}\right) p_{0}^{\prime}}} d u\right)^{1 / p_{0}^{\prime}} \\
& \times\left(\int_{2^{k+1} r_{i} \leq\left|u_{i}^{-1} u\right|<2^{k+2} r_{i}}\left|\mathscr{I}_{1}\left(V \chi_{B\left(u_{i} 2^{k+3} r_{i}\right)}\right)(u)\right|^{p_{0}} d u\right)^{1 / p_{0}} \\
& \leq\left(\int_{2^{k+1} r_{i} \leq u_{i}^{-1} u \mid<2^{k+2} r_{i}} \frac{1}{\left|u_{i}^{-1} u\right|^{\left(Q-1+\delta^{\prime}\right) p_{0}^{\prime}}} d u\right)^{1 / p_{0}^{\prime}}\left\|\mathscr{F}_{1}\left(V \chi_{B\left(u_{i} i^{2 k+3} r_{i}\right)}\right)\right\|_{L^{p_{0}}} \\
& \leq C \cdot \frac{\left|B\left(u_{i}, 2^{k+2} r_{i}\right)\right|^{1 / p_{0}^{\prime}}}{\left(2^{k+1} r_{i}\right)^{Q-1+\delta^{\prime}}}\left(\int_{B\left(u_{i} 2^{k+3} r_{i}\right)} V(w)^{q} d w\right)^{1 / q} \\
& \leq C \cdot \frac{\left|B\left(u_{i}, 2^{k+2} r_{i}\right)\right|^{1 / p_{0}^{\prime}}}{\left(2^{k+1} r_{i}\right)^{Q-1+\delta^{\prime}}}\left(\frac{1}{\left|B\left(u_{i}, 2^{k+3} r_{i}\right)\right|^{1 / q^{\prime}}} \int_{B\left(u_{i} i^{k+3} r_{i}\right)} V(w) d w\right),
\end{aligned}
$$

where the last inequality is obtained by the hypothesis $V \epsilon$ $\mathscr{B}_{q}$. Moreover, in view of (10), (105), and (57), we can see that the above expression (132) is bounded by

$$
\begin{gathered}
\frac{\left|B\left(u_{i}, 2^{k+2} r_{i}\right)\right|^{1 / p_{0}^{\prime}}}{\left(2^{k+1} r_{i}\right)^{1+\delta^{\prime}}} \cdot \frac{1}{\left|B\left(u_{i}, 2^{k+3} r_{i}\right)\right|^{1 / q^{\prime}}}\left[1+\frac{2^{k+3} r_{i}}{\rho\left(u_{i}\right)}\right]^{N_{1}} \\
\leq C \cdot \frac{1}{\left(2^{k+1} r_{i}\right)^{\delta^{\prime}}} \frac{\left|B\left(u_{i}, 2^{k+1} r_{i}\right)\right|^{1 / p_{0}^{\prime}}}{\left|B\left(u_{i}, 2^{k+1} r_{i}\right)\right|^{1 / Q}} \cdot \frac{1}{\left|B\left(u_{i}, 2^{k+1} r_{i}\right)\right|^{1 / q^{\prime}}} \\
\times\left[1+\frac{2^{k+1} r_{i}}{\rho\left(u_{i}\right)}\right]^{N_{1}}=C \cdot \frac{1}{\left(2^{k+1} r_{i}\right)^{\delta^{\prime}}}\left[1+\frac{2^{k+1} r_{i}}{\rho\left(u_{i}\right)}\right]^{N_{1}},
\end{gathered}
$$

where the last step is due to the fact that $1 / Q+1 / p_{0}-1 / q$ $=0$. Therefore, by selecting some large enough $N$ such that $N>N_{1}$, we thus have

$$
\begin{aligned}
\mathbf{I V}^{(2)} & \leq \frac{C}{\sigma} \sum_{i} r_{i}^{\delta}\left\|b_{i}\right\|_{L^{1}} \sum_{k=1}^{\infty} \frac{1}{\left(2^{k+1} r_{i}\right)^{\delta^{\prime}}}\left[1+\frac{2^{k+1} r_{i}}{\rho\left(u_{i}\right)}\right]^{-N+N_{1}} \\
& \leq \frac{C}{\sigma} \sum_{i}\left\|b_{i}\right\|_{L^{1}} \sum_{k=1}^{\infty} \frac{1}{\left(2^{k+1}\right)^{\delta^{\prime}}} \\
& \leq \frac{C}{\sigma} \sum_{i} \sigma\left|B_{i}\right| \leq \frac{C}{\sigma}\|f\|_{L^{1}\left(\mathbb{H}^{n}\right)} .
\end{aligned}
$$

Collecting all these estimates and then taking the supremum over all $\sigma>0$, we conclude the proof of Theorem 33 .

Proof of Theorem 24. Let $p_{0}$ be a positive number such that $1 / p_{0}=1 / q-1 / Q$. To prove Theorem 24 , it is enough to prove that for each given ball $B=B\left(u_{0}, r\right)$ of $\mathbb{H}^{n}$, the following estimate

$$
\begin{aligned}
& \frac{1}{\left|B\left(u_{0}, r\right)\right|^{\kappa}} \sup _{\sigma>0} \sigma \cdot\left|\left\{u \in B\left(u_{0}, r\right):\left|\mathscr{R}_{\mathscr{L}}(f)(u)\right|>\sigma\right\}\right| \\
& \quad \leq\left[1+\frac{r}{\rho\left(u_{0}\right)}\right]^{\theta},
\end{aligned}
$$

holds true for any given $f \in L_{\rho, \theta}^{1, \kappa}\left(\mathbb{H}^{n}\right)$ with some $\theta \in(0, \infty)$ and $\kappa \in\left(0,1 / p^{\prime}{ }_{0}\right)$. Using the standard technique, we decompose the function $f$ as

$$
\left\{\begin{array}{l}
f=f_{1}+f_{2} \in L_{\rho, \theta}^{1, \kappa}\left(\mathbb{H}^{n}\right), \\
f_{1}=f \cdot \chi_{2 B}, \\
f_{2}=f \cdot \chi_{(2 B)^{\complement}} .
\end{array}\right.
$$


Then, for any fixed $\sigma>0$, we can write

$$
\begin{aligned}
& \frac{1}{\left|B\left(u_{0}, r\right)\right|^{\kappa}} \sigma \cdot\left|\left\{u \in B\left(u_{0}, r\right):\left|\mathscr{R}_{\mathscr{L}}(f)(u)\right|>\sigma\right\}\right| \\
& \leq \frac{1}{\left|B\left(u_{0}, r\right)\right|^{\kappa}} \sigma \cdot\left|\left\{u \in B\left(u_{0}, r\right):\left|\mathscr{R}_{\mathscr{L}}\left(f_{1}\right)(u)\right|>\frac{\sigma}{2}\right\}\right| \\
& \quad+\frac{1}{\left|B\left(u_{0}, r\right)\right|^{\kappa}} \sigma \cdot\left|\left\{u \in B\left(u_{0}, r\right):\left|\mathscr{R}_{\mathscr{L}}\left(f_{2}\right)(u)\right|>\frac{\sigma}{2}\right\}\right| \\
& :=L_{1}+L_{2} .
\end{aligned}
$$

Let us estimate the first term $L_{1}$. By Theorem 33, (57), and (10), we get

$$
\begin{aligned}
L_{1} & \leq C \cdot \frac{1}{|B|^{\kappa}}\left(\int_{\mathbb{H}^{n}}\left|f_{1}(u)\right| d u\right)=C \cdot \frac{1}{|B|^{\kappa}}\left(\int_{2 B}|f(u)| d u\right) \\
& \leq C\|f\|_{L_{\rho, \theta}^{1, \kappa}\left(\mathbb{H}^{n}\right)} \cdot \frac{|2 B|^{\kappa}}{|B|^{\kappa}}\left[1+\frac{2 r}{\rho\left(u_{0}\right)}\right]^{\theta} \\
& \leq C_{\theta, n}\|f\|_{L_{\rho, \theta}^{1, \kappa}\left(\mathbb{H}^{n}\right)}\left[1+\frac{r}{\rho\left(u_{0}\right)}\right]^{\theta} .
\end{aligned}
$$

As for the second term $L_{2}$, from (88) and Lemma 31, it follows that for any $u \in B\left(u_{0}, r\right)$,

$$
\begin{aligned}
\left|\mathscr{R}_{\mathscr{L}}\left(f_{2}\right)(u)\right| & =\left|\int_{(2 B)^{\complement}} \mathscr{K}(u, v) f(v) d v\right|=\left|\int_{(2 B)^{\complement}} \mathscr{K}^{*}(v, u) f(v) d v\right| \\
& \leq C_{N}[\mathbf{I I I}(u)+\mathbf{I V}(u)],
\end{aligned}
$$

where

$$
\begin{aligned}
\mathbf{I I I}(u):= & \int_{(2 B)^{\complement}}\left[1+\frac{\left|v^{-1} u\right|}{\rho(u)}\right]^{-N} \frac{1}{\left|v^{-1} u\right|^{Q}} \cdot|f(v)| d v, \\
\mathbf{I V}(u):= & \int_{(2 B)^{\complement}}\left[1+\frac{\left|v^{-1} u\right|}{\rho(u)}\right]^{-N} \frac{1}{\left|v^{-1} u\right|^{Q-1}} \\
& \cdot\left\{\int_{B\left(u,\left|v^{-1} u\right| / 4\right)} \frac{V(w)}{\left|u^{-1} w\right|^{Q-1}} d w\right\} \cdot|f(v)| d v .
\end{aligned}
$$

Thus, by (139) and Chebyshev's inequality, $L_{2}$ can be written as follows:

$$
\begin{aligned}
L_{2} \leq & \frac{2}{\left|B\left(u_{0}, r\right)\right|^{\kappa}}\left(\int_{B\left(u_{0}, r\right)}\left|\mathscr{R}_{\mathscr{L}}\left(f_{2}\right)(u)\right| d u\right) \\
\leq & \frac{1}{\left|B\left(u_{0}, r\right)\right|^{\kappa}}\left(\int_{B\left(u_{0}, r\right)}|\mathbf{I I I}(u)| d u\right)+\frac{1}{\left|B\left(u_{0}, r\right)\right|^{\kappa}} \\
& \cdot\left(\int_{B\left(u_{0}, r\right)}|\mathbf{I V}(u)| d u\right):=L_{3}+L_{4} .
\end{aligned}
$$

We now proceed exactly as we did in Proof of Theorem 19 and have the following estimate as well:

$$
L_{3} \leq C\|f\|_{L_{\rho, \theta}^{1, \kappa}\left(\mathbb{H}^{n}\right)}\left[1+\frac{r}{\rho\left(u_{0}\right)}\right]^{\theta} .
$$

Let us analyze the latter term $L_{4}$. In order to do this, we first observe that

$$
\frac{1}{2}\left|v^{-1} u_{0}\right| \leq\left|v^{-1} u\right| \leq \frac{3}{2}\left|v^{-1} u_{0}\right|
$$

whenever $u \in B\left(u_{0}, r\right)$ and $v \in(2 B)^{\complement}$. Hence, for any positive integer $N \in \mathbb{N}$,

$$
\begin{aligned}
& |\mathbf{I V}(u)| \lesssim \int_{(2 B)^{\complement}}\left[1+\frac{\left|v^{-1} u_{0}\right|}{\rho(u)}\right]^{-N} \frac{1}{\left|v^{-1} u_{0}\right|^{Q-1}} \\
& \cdot\left|\int_{B\left(u,\left|v^{-1} u\right| / 4\right)} \frac{V(w)}{\left|u^{-1} w\right|^{Q-1}} d w\right| \cdot|f(v)| d v \\
& =\sum_{k=1}^{\infty} \int_{2^{k} r \leq\left|v^{-1} u_{0}\right|<2^{k+1} r}\left[1+\frac{\left|v^{-1} u_{0}\right|}{\rho(u)}\right]^{-N} \frac{1}{\left|v^{-1} u_{0}\right|^{Q-1}} \\
& \times\left|\int_{B\left(u,\left|v^{-1} u\right| / 4\right)} \frac{V(w)}{\left|u^{-1} w\right|^{Q-1}} d w\right| \cdot|f(v)| d v \\
& \lesssim \sum_{k=1}^{\infty} \frac{1}{\left|B\left(u_{0}, 2^{k+1} r\right)\right|^{1-1 / Q}} \int_{B\left(u_{0}, 2^{k+1} r\right)}\left[1+\frac{2^{k} r}{\rho(u)}\right]^{-N} \\
& \times\left|\int_{B\left(u,\left|v^{-1} u\right| / 4\right)} \frac{V(w)}{\left|u^{-1} w\right|^{Q-1}} d w\right| \cdot|f(v)| d v .
\end{aligned}
$$

It is easy to verify that when $w \in B\left(u,\left|v^{-1} u\right| / 4\right)$ and $v$ $\in B\left(u_{0}, 2^{k+1} r\right)$, one has $w \in B\left(u_{0}, 2^{k+2} r\right)$. This fact together with (107) implies that for any $u \in B\left(u_{0}, r\right)$,

$$
\begin{aligned}
& |\mathbf{I V}(u)| \lesssim \sum_{k=1}^{\infty} \frac{1}{\left|B\left(u_{0}, 2^{k+1} r\right)\right|^{1-1 / Q}}\left[1+\frac{2^{k} r}{\rho(u)}\right]^{-N} \times \int_{B\left(u_{0}, 2^{k+1} r\right)} \\
& \cdot\left|\mathscr{I}_{1}\left(\left(V \chi_{B\left(u_{0}, 2^{k+2} r\right)}\right)(u)\right)\right| \cdot|f(v)| d v \\
& \lesssim \sum_{k=1}^{\infty} \frac{1}{\left|B\left(u_{0}, 2^{k+1} r\right)\right|^{1-1 / Q}}\left[1+\frac{2^{k+1} r}{\rho\left(u_{0}\right)}\right]^{-N \cdot\left(1 /\left(N_{0}+1\right)\right)} \\
& \cdot \int_{B\left(u_{0}, 2^{k+1} r\right)}|f(v)| d v \times\left|\mathscr{I}_{1}\left(\left(V \chi_{B\left(u_{0}, 2^{k+2} r\right)}\right)(u)\right)\right| \\
& \lesssim \sum_{k=1}^{\infty} \frac{\left|B\left(u_{0}, 2^{k+1} r\right)\right|^{\kappa}}{\left|B\left(u_{0}, 2^{k+1} r\right)\right|^{1-1 / Q}}\left[1+\frac{2^{k+1} r}{\rho\left(u_{0}\right)}\right]^{-N \cdot\left(1 /\left(N_{0}+1\right)\right)+\theta} \\
& \text { - }\|f\|_{L_{\rho, \theta}^{1, \kappa}\left(\mathbb{H}^{n}\right)} \times\left|\mathscr{J}_{1}\left(\left(V \chi_{B\left(u_{0}, 2^{k+2} r\right)}\right)(u)\right)\right| \text {. }
\end{aligned}
$$


Consequently,

$$
\begin{aligned}
L_{4} \leq & C\|f\|_{L_{\rho, \theta}^{1, k}\left(\mathbb{H}^{n}\right)} \frac{1}{\left|B\left(u_{0}, r\right)\right|^{k}} \sum_{k=1}^{\infty} \frac{\left|B\left(u_{0}, 2^{k+1} r\right)\right|^{\kappa}}{\left|B\left(u_{0}, 2^{k+1} r\right)\right|^{1-1 / Q}} \\
& \cdot\left[1+\frac{2^{k+1} r}{\rho\left(u_{0}\right)}\right]^{-N \cdot\left(1 /\left(N_{0}+1\right)\right)+\theta} \\
& \times\left(\int_{B\left(u_{0}, r\right)}\left|\mathscr{I}_{1}\left(\left(V \chi_{B\left(u_{0}, 2^{k+2} r\right)}\right)(u)\right)\right| d u\right) .
\end{aligned}
$$

Applying the Hölder inequality along with (103), we can compute the above integral as follows:

$$
\begin{aligned}
& \int_{B\left(u_{0}, r\right)}\left|\mathscr{I}_{1}\left(V \chi_{B\left(u_{0}, 2^{k+2} r\right)}\right)(u)\right| d u \\
& \quad \leq\left(\int_{B\left(u_{0}, r\right)} 1 d u\right)^{1 / p_{0}^{\prime}}\left(\int_{B\left(u_{0}, r\right)}\left|\mathscr{I}_{1}\left(V \chi_{B\left(u_{0}, 2^{k+2} r\right)}\right)(u)\right|^{p_{0}} d u\right)^{1 / p_{0}} \\
& \quad \leq\left|B\left(u_{0}, r\right)\right|^{1 / p_{0}^{\prime}}\left\|\mathscr{I}_{1}\left(V \chi_{B\left(u_{0}, 2^{k+2} r\right)}\right)\right\|_{L^{p_{0}}} \\
& \quad \leq C\left|B\left(u_{0}, r\right)\right|^{1 / p_{0}^{\prime}}\left(\int_{B\left(u_{0}, 2^{k+2} r\right)} V(w)^{q} d w\right)^{1 / q} \\
& \quad \leq C\left|B\left(u_{0}, r\right)\right|^{1 / p_{0}^{\prime}}\left(\frac{1}{\left|B\left(u_{0}, 2^{k+2} r\right)\right|^{1 / q^{\prime}}} \int_{B\left(u_{0}, 2^{k+2} r\right)} V(w) d w\right)
\end{aligned}
$$

where the last inequality holds since $V \in \mathscr{B}_{q}$. Moreover, by (105), (57), and (10), we can see that the above expression is bounded by

$$
\begin{aligned}
& C\left|B\left(u_{0}, r\right)\right|^{1 / p_{0}^{\prime}} \cdot \frac{\left(2^{k+2} r\right)^{Q-2}}{\left|B\left(u_{0}, 2^{k+2} r\right)\right|^{1 / q^{\prime}}}\left[1+\frac{2^{k+2} r}{\rho\left(u_{0}\right)}\right]^{N_{1}} \\
& \leq C\left|B\left(u_{0}, r\right)\right|^{1 / p_{0}^{\prime}} \cdot \frac{\left(2^{k+1} r\right)^{Q-2}}{\left|B\left(u_{0}, 2^{k+1} r\right)\right|^{1 / q^{\prime}}}\left[1+\frac{2^{k+1} r}{\rho\left(u_{0}\right)}\right]^{N_{1}} .
\end{aligned}
$$

Taking into account the fact that $1 / Q+1 / q^{\prime}=1 / p^{\prime}{ }_{0}$, then we have

$$
\begin{aligned}
L_{4} \leq & C\|f\|_{L_{\rho, \theta}^{1, \kappa}\left(\mathbb{H}^{n}\right)}\left|B\left(u_{0}, r\right)\right|^{1 / p_{0}^{\prime}-\kappa} \sum_{k=1}^{\infty} \frac{\left|B\left(u_{0}, 2^{k+1} r\right)\right|^{\kappa}}{\left|B\left(u_{0}, 2^{k+1} r\right)\right|^{1-1 / Q+1 / q^{\prime}-1+2 / Q}} \\
& \cdot\left[1+\frac{2^{k+1} r}{\rho\left(u_{0}\right)}\right]^{-N \cdot\left(1 /\left(N_{0}+1\right)\right)+\theta+N_{1}} \\
= & C\|f\|_{L_{\rho, \theta}^{1, \kappa}\left(\mathbb{H}^{n}\right)} \sum_{k=1}^{\infty} \frac{\left|B\left(u_{0}, r\right)\right|^{1 / p_{0}^{\prime}-\kappa}}{\left|B\left(u_{0}, 2^{k+1} r\right)\right|^{1 / p_{0}^{\prime}-\kappa}}\left[1+\frac{2^{k+1} r}{\rho\left(u_{0}\right)}\right]^{-N \cdot\left(1 /\left(N_{0}+1\right)\right)+\theta+N_{1}} \\
\leq & C\|f\|_{L_{\rho, \theta}^{1, \kappa}\left(\mathbb{H}^{n}\right)} \sum_{k=1}^{\infty} \frac{\left|B\left(u_{0}, r\right)\right|^{1 / p_{0}^{\prime}-\kappa}}{\left|B\left(u_{0}, 2^{k+1} r\right)\right|^{1 / p_{0}^{\prime}-\kappa}},
\end{aligned}
$$

where a large enough $N$ is chosen satisfying $N>\left(N_{0}+1\right)$ $\left(\theta+N_{1}\right)$. By the choice of $\kappa$, it guarantees that the exponent $-\left(1 / p^{\prime}{ }_{0}-\kappa\right)$ of the last summation is negative, and hence, it is convergent. Therefore, we conclude that

$$
L_{4} \leq C\|f\|_{L_{\rho, \theta}^{1, \kappa}\left(\mathbb{H}^{n}\right)} \leq C\|f\|_{L_{\rho, \theta}^{1, \kappa}\left(\mathbb{H}^{n}\right)}\left[1+\frac{r}{\rho\left(u_{0}\right)}\right]^{\theta}
$$

Combining these estimates for $L_{1}, L_{3}$, and $L_{4}$, and then taking the supremum over all $\sigma>0$, we get the desired estimate (135). This finishes Proof of Theorem 24.

Proof of Theorem 23. Since the proof is similar to that of Theorem 22, we shall only indicate the necessary modifications. As before, it is enough for us to show that for an arbitrary fixed ball $B\left(u_{0}, r\right)$ in $\mathbb{H}^{n}$, the following estimate

$$
\left(\frac{1}{\left|B\left(u_{0}, r\right)\right|^{\kappa}} \int_{B\left(u_{0}, r\right)}\left|\mathscr{R}_{\mathscr{L}}(f)(u)\right|^{p} d u\right)^{1 / p} \lesssim\left[1+\frac{r}{\rho\left(u_{0}\right)}\right]^{\theta},
$$

holds true for any given $f \in L_{\rho, \theta}^{p, \kappa}\left(\mathbb{H}^{n}\right)$ with some $\theta \in(0, \infty)$, $p \in\left(1, p_{0}\right)$, and $\kappa \in\left(0,1 / s^{\prime}\right)$, where $s=p_{0} / p$ and $1 / p_{0}=1 / p-$ $1 / Q$. To this end, we split $f=f_{1}+f_{2}$ through $f_{1}=f \cdot \chi_{2 B}$ and $f_{2}=f \cdot \chi_{(2 B)^{c}}$. Then, the left-hand side of (151) will be divided into two parts given below.

$$
\begin{aligned}
& \left(\frac{1}{\left|B\left(u_{0}, r\right)\right|^{\kappa}} \int_{B\left(u_{0}, r\right)}\left|\mathscr{R}_{\mathscr{L}}(f)(u)\right|^{p} d u\right)^{1 / p} \\
& \leq\left(\frac{1}{\left|B\left(u_{0}, r\right)\right|^{\kappa}} \int_{B\left(u_{0}, r\right)}\left|\mathscr{R}_{\mathscr{L}}\left(f_{1}\right)(u)\right|^{p} d u\right)^{1 / p} \\
& \quad+\left(\frac{1}{\left|B\left(u_{0}, r\right)\right|^{\kappa}} \int_{B\left(u_{0}, r\right)}\left|\mathscr{R}_{\mathscr{L}}\left(f_{2}\right)(u)\right|^{p} d u\right)^{1 / p}:=M_{1}+M_{2} .
\end{aligned}
$$

Since the Riesz transform $\mathscr{R}_{\mathscr{L}}$ is bounded on $L^{p}\left(\mathbb{H}^{n}\right)$ for $1<p<p_{0}$ (see Theorem 12), we can deal with $M_{1}$ in the same manner as in Proof of Theorem 22 and obtain

$$
M_{1} \leq C\|f\|_{L_{\rho, \theta}^{p, \kappa}\left(\mathbb{H}^{n}\right)}\left[1+\frac{r}{\rho\left(u_{0}\right)}\right]^{\theta}
$$

On the other hand, from (139), it follows that

$$
\begin{aligned}
M_{2} \lesssim & \left(\frac{1}{\left|B\left(u_{0}, r\right)\right|^{\kappa}} \int_{B\left(u_{0}, r\right)}|\mathbf{I I I}(u)|^{p} d u\right)^{1 / p} \\
& +\left(\frac{1}{\left|B\left(u_{0}, r\right)\right|^{\kappa}} \int_{B\left(u_{0}, r\right)}|\mathbf{I V}(u)|^{p} d u\right)^{1 / p}:=M_{3}+M_{4} .
\end{aligned}
$$


Here

$$
\begin{aligned}
\operatorname{III}(u)= & \int_{(2 B)^{\complement}}\left[1+\frac{\left|v^{-1} u\right|}{\rho(u)}\right]^{-N} \frac{1}{\left|v^{-1} u\right|^{Q}} \cdot|f(v)| d v, \\
\mathbf{I V}(u)= & \int_{(2 B)^{\complement}}\left[1+\frac{\left|v^{-1} u\right|}{\rho(u)}\right]^{-N} \frac{1}{\left|v^{-1} u\right|^{Q-1}} \\
& \cdot\left\{\int_{B\left(u,\left|v^{-1} u\right| / 4\right)} \frac{V(w)}{\left|u^{-1} w\right|^{Q-1}} d w\right\} \cdot|f(v)| d v .
\end{aligned}
$$

We follow the same arguments as in Proof of Theorem 18 and obtain the following estimate as well:

$$
M_{3} \leq C\|f\|_{L_{\rho, \theta}^{p, \kappa}\left(\mathbb{H}^{n}\right)}\left[1+\frac{r}{\rho\left(u_{0}\right)}\right]^{\theta} .
$$

It remains to check that (151) holds for the last term $M_{4}$. As it was shown in Theorem 24, it holds that for any $u \in B\left(u_{0}, r\right)$,

$$
\begin{aligned}
|\mathbf{I V}(u)| \lesssim & \sum_{k=1}^{\infty} \frac{1}{\left|B\left(u_{0}, 2^{k+1} r\right)\right|^{1-1 / Q}}\left[1+\frac{2^{k} r}{\rho(u)}\right]^{-N} \\
& \times \int_{B\left(u_{0}, 2^{k+1} r\right)}\left|\mathscr{I}_{1}\left(V \chi_{B\left(u_{0}, 2^{k+2} r\right)}\right)(u)\right| \cdot|f(v)| d v \\
\lesssim & \sum_{k=1}^{\infty} \frac{1}{\left|B\left(u_{0}, 2^{k+1} r\right)\right|^{1-1 / Q}}\left[1+\frac{2^{k+1} r}{\rho\left(u_{0}\right)}\right]^{-N \cdot\left(1 /\left(N_{0}+1\right)\right)} \\
& \cdot \int_{B\left(u_{0}, 2^{k+1} r\right)}|f(v)| d v \times\left|\mathscr{I}_{1}\left(V \chi_{B\left(u_{0}, 2^{k+2} r\right)}\right)(u)\right| .
\end{aligned}
$$

Moreover, by using the Hölder inequality, we obtain that for each fixed $k \in \mathbb{N}$,

$$
\begin{aligned}
\int_{B\left(u_{0}, 2^{k+1} r\right)}|f(v)| d v \leq & \left(\int_{B\left(u_{0}, 2^{k+1} r\right)}|f(v)|^{\mathrm{p}} d v\right)^{1 / p} \\
& \cdot\left(\int_{B\left(u_{0}, 2^{k+1} r\right)} 1 d v\right)^{1 / p^{\prime}} \\
\leq & C\|f\|_{L_{\rho, \theta}^{p, \kappa}\left(\mathbb{H}^{n}\right)}\left|B\left(u_{0}, 2^{k+1} r\right)\right|^{\kappa / p}\left[1+\frac{2^{k+1} r}{\rho\left(u_{0}\right)}\right]^{\theta} \\
& \cdot\left|B\left(u_{0}, 2^{k+1} r\right)\right|^{1 / p^{\prime}},
\end{aligned}
$$

which in turn gives

$$
\begin{aligned}
M_{4} \leq & C\|f\|_{L_{\rho, \theta}^{p, \kappa}\left(\mathbb{H}^{n}\right)} \frac{1}{\left|B\left(u_{0}, r\right)\right|^{\kappa / p}} \sum_{k=1}^{\infty} \frac{\left|B\left(u_{0}, 2^{k+1} r\right)\right|^{\kappa / p}}{\left|B\left(u_{0}, 2^{k+1} r\right)\right|^{1 / p-1 / Q}} \\
& \cdot\left[1+\frac{2^{k+1} r}{\rho\left(u_{0}\right)}\right]^{-N \cdot\left(1 /\left(N_{0}+1\right)\right)+\theta} \\
\times & \left(\int_{B\left(u_{0}, r\right)}\left|\mathscr{I}_{1}\left(V \chi_{B\left(u_{0}, 2^{k+2} r\right)}\right)(u)\right|^{p} d u\right)^{1 / p} .
\end{aligned}
$$

For the latter integral, we use the Hölder inequality with exponent $s=p_{0} / p>1$ and (103) to derive

$$
\begin{aligned}
& \left(\int_{B\left(u_{0}, r\right)}\left|\mathscr{I}_{1}\left(V \chi_{B\left(u_{0}, 2^{k+2} r\right)}\right)(u)\right|^{p} d u\right)^{1 / p} \\
& \quad \leq\left(\int_{B\left(u_{0}, r\right)} 1 d u\right)^{1 /\left(p s^{\prime}\right)}\left(\int_{B\left(u_{0}, r\right)}\left|\mathscr{I}_{1}\left(V \chi_{B\left(u_{0}, 2^{k+2} r\right)}\right)(u)\right|^{p_{0}} d u\right)^{1 / p_{0}} \\
& \quad \leq\left|B\left(u_{0}, r\right)\right|^{1 /\left(p s^{\prime}\right)} \mid\left\|\mathscr{I}_{1}\left(V \chi_{B\left(u_{0}, 2^{k+2} r\right)}\right)\right\|_{L^{p_{0}}} \\
& \quad \leq C\left|B\left(u_{0}, r\right)\right|^{1 /\left(p s^{\prime}\right)}\left(\int_{B\left(u_{0}, 2^{k+2} r\right)} V(w)^{q} d \mathrm{w}\right)^{1 / q} \\
& \quad \leq C\left|B\left(u_{0}, r\right)\right|^{1 /\left(p s^{\prime}\right)}\left(\frac{1}{\left|B\left(u_{0}, 2^{k+2} r\right)\right|^{1 / q^{\prime}}} \int_{B\left(u_{0}, 2^{k+2} r\right)} V(w) d w\right)
\end{aligned}
$$

where the last inequality is obtained by the fact that $V \in$ $\mathscr{B}_{q}$. Furthermore, in view of (105), (57), and (10), we can see that the above expression is controlled by

$$
\begin{gathered}
C\left|B\left(u_{0}, r\right)\right|^{1 /\left(p s^{\prime}\right)} \cdot \frac{\left(2^{k+2} r\right)^{Q-2}}{\left|B\left(u_{0}, 2^{k+2} r\right)\right|^{1 / q^{\prime}}}\left[1+\frac{2^{k+2} r}{\rho\left(u_{0}\right)}\right]^{N_{1}} \\
\leq C\left|B\left(u_{0}, r\right)\right|^{1 /\left(p s^{\prime}\right)} \cdot \frac{\left(2^{k+1} r\right)^{Q-2}}{\left|B\left(u_{0}, 2^{k+1} r\right)\right|^{1 / q^{\prime}}}\left[1+\frac{2^{k+1} r}{\rho\left(u_{0}\right)}\right]^{N_{1}} .
\end{gathered}
$$

A trivial computation leads to

$$
\frac{1}{p}-\frac{1}{Q}+\frac{1}{q^{\prime}}-1+\frac{2}{Q}=\frac{1}{p}-\frac{1}{p_{0}}=\frac{1}{\left(p s^{\prime}\right)}
$$


Taking into account this fact, then we have

$$
\begin{aligned}
M_{4} \leq & C\|f\|_{L_{\rho, \theta}^{p, k}\left(\mathbb{H}^{n}\right)}\left|B\left(u_{0}, r\right)\right|^{1 /\left(p s^{\prime}\right)-\kappa / p} \\
& \times \sum_{k=1}^{\infty} \frac{\left|B\left(u_{0}, 2^{k+1} r\right)\right|^{\kappa / p}}{\left|B\left(u_{0}, 2^{k+1} r\right)\right|^{1 / p-1 / Q+1 / q^{\prime}-1+2 / Q}} \\
& \cdot\left[1+\frac{2^{k+1} r}{\rho\left(u_{0}\right)}\right]^{-N \cdot\left(1 /\left(N_{0}+1\right)\right)+\theta+N_{1}} \\
= & C\|f\|_{L_{\rho, \theta}^{p, k}\left(\mathbb{H}^{n}\right)} \sum_{k=1}^{\infty} \frac{\left|B\left(u_{0}, r\right)\right|^{\left(1 / s^{\prime}-\kappa\right) / p}}{\left|B\left(u_{0}, 2^{k+1} r\right)\right|^{\left(1 / s^{\prime}-\kappa\right) / p}} \\
& \cdot\left[1+\frac{2^{k+1} r}{\rho\left(u_{0}\right)}\right]^{-N \cdot\left(1 /\left(N_{0}+1\right)\right)+\theta+N_{1}} \\
\leq & C\|f\|_{L_{\rho, \theta}^{p, k}\left(\mathbb{H}^{n}\right)} \sum_{k=1}^{\infty} \frac{\left|B\left(u_{0}, r\right)\right|^{\left(1 / s^{\prime}-\kappa\right) / p}}{\left|B\left(u_{0}, 2^{k+1} r\right)\right|^{\left(1 / s^{\prime}-\kappa\right) / p}},
\end{aligned}
$$

where a large enough $N$ is chosen such that $N>\left(N_{0}+1\right)$ $\left(\theta+N_{1}\right)$. By the hypothesis, we know that the exponent in the last summation is negative, and hence, it is convergent. Therefore, we conclude that

$$
M_{4} \leq C\|f\|_{L_{\rho, \theta}^{p, \kappa}\left(\mathbb{H}^{n}\right)} \leq C\|f\|_{L_{\rho, \theta}^{p, \kappa}\left(\mathbb{H}^{n}\right)}\left[1+\frac{r}{\rho\left(u_{0}\right)}\right]^{\theta},
$$

as desired. This finishes Proof of Theorem 23.

If one has a slightly stronger assumption on the potential $V$, then we have the following improved estimates for the kernels $\mathscr{K}(u, v)$ and $\mathscr{K}^{*}(u, v)$.

Lemma 34. Let $V \in \mathscr{B}_{q}$ with $q \in[Q, \infty)$, and let $\rho(\cdot)$ be the auxiliary function determined by $V$. For every positive integer $N \in \mathbb{N}$, there exists a positive constant $C_{N}>0$ such that, for any $u$ and $v$ in $\mathbb{H}^{n}$,

$$
\left\{\begin{array}{l}
|\mathscr{K}(u, v)| \leq C_{N}\left[1+\frac{\left|v^{-1} u\right|}{\rho(u)}\right]^{-N} \frac{1}{\left|v^{-1} u\right|^{Q}}, \\
\left|\mathscr{K}^{*}(u, v)\right| \leq C_{N}\left[1+\frac{\left|v^{-1} u\right|}{\rho(u)}\right]^{-N} \frac{1}{\left|v^{-1} u\right|^{Q}} .
\end{array}\right.
$$

We remark that in the Euclidean case, this lemma was already obtained by Shen in [10]. Moreover, Shen [10] actually showed that $\mathscr{R}_{\mathscr{L}}$ and its dual form $\mathscr{R}_{\mathscr{L}}^{*}$ are standard Calderón-Zygmund singular integral operators in $\mathbb{R}^{d}$, and hence, these two operators $\mathscr{R}_{\mathscr{L}}$ and $\mathscr{R}_{\mathscr{L}}^{*}$ are all bounded on $L^{p}\left(\mathbb{R}^{d}\right)$ for $1<p<\infty$ and are of weak-type $(1,1)$, when $V$ $\in \mathscr{B}_{q}$ with $d \leq q<\infty$. We adapt the arguments used in [10, 42] (see also $[19,21]$ ) to our present situation and prove Lemma 34 similarly. Furthermore, by adopting the same method given in $[3,10]$, we can also prove that the operators $\mathscr{R}_{\mathscr{L}}$ and $\mathscr{R}_{\mathscr{L}}^{*}$ are bounded on $L^{p}\left(\mathbb{H}^{n}\right)$ for all $1<p<\infty$ and are bounded from $L^{1}\left(\mathbb{H}^{n}\right)$ into $W L^{1}\left(\mathbb{H}^{n}\right)$ in such a situation.
Repeating the arguments above, we are able to show that under the same assumptions as in Theorems 18 and 19, the corresponding results also hold for the operators $\mathscr{R}_{\mathscr{L}}$ and $\mathscr{R}_{\mathscr{L}}^{*}$ on $\mathbb{H}^{n}$.

Theorem 35. Let $0<\kappa<1$ and $0<\theta<\infty$. If $V \in \mathscr{B}_{q}$ with $q$ $\in[Q, \infty)$, then the operators $\mathscr{R}_{\mathscr{L}}^{*}$ and $\mathscr{R}_{\mathscr{L}}$ are bounded linear operators on $L_{\rho, \theta}^{p, \kappa}\left(\mathbb{H}^{n}\right)$ for all $1<p<\infty$ and hence bounded on $L_{\rho, \infty}^{p, \kappa}\left(\mathbb{H}^{n}\right)$.

Theorem 36. Let $0<\kappa<1$ and $0<\theta<\infty$. If $V \in \mathscr{B}_{q}$ with $q$ $\in[Q, \infty)$, then the operators $\mathscr{R}_{\mathscr{L}}^{*}$ and $\mathscr{R}_{\mathscr{L}}$ are bounded linear operators from $L_{\rho, \theta}^{1, \kappa}\left(\mathbb{H}^{n}\right)$ into $W L_{\rho, \theta}^{1, \kappa}\left(\mathbb{H}^{n}\right)$ and hence bounded from $L_{\rho, \infty}^{1, \kappa}\left(\mathbb{H}^{n}\right)$ into $W L_{\rho, \infty}^{1, \kappa}\left(\mathbb{H}^{n}\right)$.

We recall the relation $1 / p_{0}=1 / p-1 / Q$. Since $p_{0}$ tends to $\infty$ as $q \rightarrow Q$, so we have the following: $\left(p_{0}\right)^{\prime}$ tends to 1 , and $s^{\prime}$ tends to 1 with $s=p_{0} / p$. Hence, the above theorems can be regarded as the limiting case of the results of Theorems 22,23 , and 24 .

\section{Generalized Morrey Spaces}

In the last section, let us give the definitions of the generalized Morrey spaces related to the nonnegative potential $V$ on $\mathbb{H}^{n}$. Let $\Phi=\Phi(r), r>0$, be a growth function, that is, a positive increasing function on $(0, \infty)$, and satisfy the following doubling condition:

$$
\Phi(2 r) \leq D \Phi(r)
$$

for all $r>0$, where $D=D(\Phi) \geq 1$ is a doubling constant independent of $r$.

Definition 37. Let $\rho(\cdot)$ be the auxiliary function determined by $V \in \mathscr{B}_{q}$ with $q \in[Q / 2, \infty)$. Let $1 \leq p<\infty$ and $\Phi$ be a growth function. For any given $0<\theta<\infty$, the generalized Morrey space $L_{\rho, \theta}^{p, \Phi}\left(\mathbb{H}^{n}\right)$ is defined to be the set of all $p$ -locally integrable functions $f$ on $\mathbb{H}^{n}$ such that

$$
\left(\frac{1}{\Phi\left(\left|B\left(u_{0}, r\right)\right|\right)} \int_{B\left(u_{0}, r\right)}|f(u)|^{p} d u\right)^{1 / p} \leq C \cdot\left[1+\frac{r}{\rho\left(u_{0}\right)}\right]^{\theta},
$$

holds for every ball $B\left(u_{0}, r\right)$ in $\mathbb{H}^{n}$, and we denote the smallest constant $C$ satisfying (167) by $\|f\|_{L_{\rho, \theta}^{p, \Phi}\left(\mathbb{H}^{n}\right)}$. It is easy to see that the functional $\|\cdot\|_{L_{\rho, \theta}^{p, \Phi}\left(\mathbb{H}^{n}\right)}$ is a norm on the linear space $L_{\rho, \theta}^{p, \Phi}\left(\mathbb{H}^{n}\right)$ that makes it into a Banach space under this norm. Define

$$
L_{\rho, \infty}^{p, \Phi}\left(\mathbb{H}^{n}\right):=\bigcup_{0<\theta<\infty} L_{\rho, \theta}^{p, \Phi}\left(\mathbb{H}^{n}\right)
$$


Definition 38. Let $\rho(\cdot)$ be the auxiliary function determined by $V \in \mathscr{B}_{q}$ with $q \in[Q / 2, \infty)$. Let $p=1$ and $\Phi$ be a growth function. For any given $0<\theta<\infty$, the generalized weak Morrey space $W L_{\rho, \theta}^{1, \Phi}\left(\mathbb{H}^{n}\right)$ is defined to be the set of all measurable functions $f$ on $\mathbb{H}^{n}$ such that

$$
\begin{aligned}
& \frac{1}{\Phi\left(\left|B\left(u_{0}, r\right)\right|\right)} \sup _{\lambda>0} \lambda \cdot\left|\left\{u \in B\left(u_{0}, r\right):|f(u)|>\lambda\right\}\right| \\
& \quad \leq C \cdot\left[1+\frac{r}{\rho\left(u_{0}\right)}\right]^{\theta},
\end{aligned}
$$

holds for every ball $B\left(u_{0}, r\right)$ in $\mathbb{H}^{n}$, and we denote the smallest constant $C$ satisfying (169) by $\|f\|_{W L_{\rho, \theta}^{1, \Phi}\left(\mathbb{H}^{n}\right)}$. Correspondingly, we define

$$
W L_{\rho, \infty}^{1, \Phi}\left(\mathbb{H}^{n}\right):=\bigcup_{0<\theta<\infty} W L_{\rho, \theta}^{1, \Phi}\left(\mathbb{H}^{n}\right) .
$$

Remark 39.

(i) As in Section 2 (Remark 17), we can also define a norm and a (quasi-)norm on the linear spaces $L_{\rho, \infty}^{p, \Phi}$ $\left(\mathbb{H}^{n}\right)$ and $W L_{\rho, \infty}^{1, \Phi}\left(\mathbb{H}^{n}\right)$, respectively

(ii) According to this definition, we recover the spaces $L_{\rho, \theta}^{p, \kappa}\left(\mathbb{H}^{n}\right)$ and $W L_{\rho, \theta}^{1, \kappa}\left(\mathbb{H}^{n}\right)$ under the choice $\Phi(t)=$ $t^{\kappa}$, for all $(p, \kappa) \in[1, \infty) \times(0,1)$

(iii) In the Euclidean setting, when $\theta=0$ or $V \equiv 0$, the classes $L_{\rho, \theta}^{p, \Phi}$ and $W L_{\rho, \theta}^{1, \Phi}$ reduce to the classes $L^{p, \Phi}$ and $W L^{1, \Phi}$, which were introduced and studied by Mizuhara in [45]. We refer the reader to [46, 47] for further details

By using the same procedure as in the proofs of our main results, we have the following theorems. We leave the details to the reader. Let $\rho$ be as in (14).

Theorem 40. Let $1<p<\infty, 1 \leq D(\Phi)<2$, and $0<\theta<\infty$. If $V \in \mathscr{B}_{q}$ with $q \in[Q, \infty)$, then the operators $\mathscr{R}_{\mathscr{L}}^{*}$ and $\mathscr{R}_{\mathscr{L}}$ are bounded on $L_{\rho, \theta}^{p, \Phi}\left(\mathbb{H}^{n}\right)$ and hence bounded on $L_{\rho, \infty}^{p, \Phi}\left(\mathbb{H}^{n}\right)$.

Theorem 41. Let $p=1,1 \leq D(\Phi)<2$, and $0<\theta<\infty$. If $V \in$ $\mathscr{B}_{q}$ with $q \in[Q, \infty)$, then the operators $\mathscr{R}_{\mathscr{L}}^{*}$ and $\mathscr{R}_{\mathscr{L}}$ are bounded from $L_{\rho, \theta}^{1, \Phi}\left(\mathbb{H}^{n}\right)$ into $W L_{\rho, \theta}^{1, \Phi}\left(\mathbb{H}^{n}\right)$ and hence bounded from $L_{\rho, \infty}^{1, \Phi}\left(\mathbb{H}^{n}\right)$ into $W L_{\rho, \infty}^{1, \Phi}\left(\mathbb{H}^{n}\right)$.

We point out that the same conclusions also hold for $\mathscr{T}_{\mathscr{L}}^{*}$ and other maximal functions $\left(\mathscr{T}_{\sqrt{\mathscr{L}}}^{*}, \mathscr{T}_{\mathscr{L}}^{* *}\right.$, and $\left.\mathscr{T}_{\sqrt{\mathscr{L}}}^{* *}\right)$ discussed in Section 3.

Theorem 42. Let $1 \leq D(\Phi)<2$ and $0<\theta<\infty$. If $V \in \mathscr{B}_{q}$ with $q \in[Q / 2, Q)$, and $p_{0}$ is a number such that $1 / p_{0}=1 / q-1 / Q$, then the operator $\mathscr{R}_{\mathscr{L}}^{*}$ is bounded on $L_{\rho, \theta}^{p, \Phi}\left(\mathbb{H}^{n}\right)$ and hence bounded on $L_{\rho, \infty}^{p, \Phi}\left(\mathbb{H}^{n}\right)$ provided that $\left(p_{0}\right)^{\prime}<p<\infty$.

Theorem 43. Let $0<\theta<\infty$. If $V \in \mathscr{B}_{q}$ with $q \in[Q / 2, Q)$, and $p_{0}$ is a number such that $1 / p_{0}=1 / q-1 / Q$, then the operator $\mathscr{R}_{\mathscr{L}}$ is bounded on $L_{\rho, \theta}^{p, \Phi}\left(\mathbb{H}^{n}\right)$ and hence bounded on $L_{\rho, \infty}^{p, \Phi}$ $\left(\mathbb{H}^{n}\right)$ provided that $1<p<p_{0}$ and $1 \leq D(\Phi)<2^{1 / s^{\prime}}$ with $s:=p_{0} / p$.

Theorem 44. Let $0<\theta<\infty$. If $V \in \mathscr{B}_{q}$ with $q \in[Q / 2, Q)$, and $p_{0}$ is a number such that $1 / p_{0}=1 / q-1 / Q$, then the operator $\mathscr{R}_{\mathscr{L}}$ is bounded from $L_{\rho, \theta}^{1, \Phi}\left(\mathbb{H}^{n}\right)$ into $W L_{\rho, \theta}^{1, \Phi}\left(\mathbb{H}^{n}\right)$ and hence bounded from $L_{\rho, \infty}^{1, \Phi}\left(\mathbb{H}^{n}\right)$ into $W L_{\rho, \infty}^{1, \Phi}\left(\mathbb{H}^{n}\right)$ provided that $1 \leq$ $D(\Phi)<2^{1 /\left(p_{0}\right)^{\prime}}$.

\section{Data Availability}

No data were used to support this study.

\section{Conflicts of Interest}

The author declares that there is no conflict of interest regarding the publication of this paper.

\section{References}

[1] G. B. Folland and E. M. Stein, "Estimates for the complex and analysis on the Heisenberg group," Communications on Pure and Applied Mathematics, vol. 27, no. 4, pp. 429-522, 1974.

[2] E. M. Stein, Harmonic Analysis: Real-Variable Methods, Orthogonality, and Oscillatory Integrals, Princeton University Press, Princeton, NJ, USA, 1993.

[3] C.-C. Lin and H. Liu, "BMO $O_{\mathrm{L}}\left(\mathbb{H}^{n}\right)$ spaces and Carleson measures for Schrödinger operators," Advances in Mathematics, vol. 228, no. 3, pp. 1631-1688, 2011.

[4] G. B. Folland, Harmonic Analysis in Phase Space, Annals of Mathematics Studies, Princeton University Press, Princeton, NJ, USA, 1989.

[5] S. Thangavelu, Harmonic Analysis on the Heisenberg Group, Progress in Mathematics, vol. 159, Birkhauser, Boston/Basel/Berlin, 1998.

[6] J. Dziubański and J. Zienkiewicz, "Hardy spaces associated with some Schrödinger operators," Studia Mathematica, vol. 126, no. 2, pp. 149-160, 1997.

[7] J. Dziubański and J. Zienkiewicz, "Hardy space $\mathrm{H}^{1}$ associated to Schrödinger operator with potential satisfying reverse Hölder inequality," Revista Matemática Iberoamericana, vol. 15, pp. 279-296, 1999.

[8] J. Dziubański and J. Zienkiewicz, "Hpspaces associated with Schrödinger operators with potentials from reverse Hölder classes," Colloquium Mathematicum, vol. 98, no. 1, pp. 5-38, 2003.

[9] J. Dziubański, G. Garrigós, T. Martínez, J. L. Torrea, and J. Zienkiewicz, "BMO spaces related to Schrödinger operators with potentials satisfying a reverse Hölder inequality," Mathematische Zeitschrift, vol. 249, no. 2, pp. 329-356, 2005. 
[10] Z. W. Shen, " $\mathrm{L}^{\mathrm{p}}$ estimates for Schrödinger operators with certain potentials," Annales de l'institut Fourier, vol. 45, no. 2, pp. 513-546, 1995.

[11] B. Bongioanni, E. Harboure, and O. Salinas, "Classes of weights related to Schrödinger operators," Journal of Mathematical Analysis and Applications, vol. 373, no. 2, pp. 563-579, 2011.

[12] B. Bongioanni, E. Harboure, and O. Salinas, "Weighted inequalities for commutators of Schrödinger-Riesz transforms," Journal of Mathematical Analysis and Applications, vol. 392, no. 1, pp. 6-22, 2012.

[13] B. Bongioanni, A. Cabral, and E. Harboure, "Extrapolation for classes of weights related to a family of operators and applications," Potential Analysis, vol. 38, no. 4, pp. 1207-1232, 2013.

[14] B. Bongioanni, A. Cabral, and E. Harboure, "Lerner's inequality associated to a critical radius function and applications," Journal of Mathematical Analysis and Applications, vol. 407, no. 1, pp. 35-55, 2013.

[15] T. A. Bui, "Weighted estimates for commutators of some singular integrals related to Schrödinger operators," Bulletin des Sciences Mathematiques, vol. 138, no. 2, pp. 270-292, 2014.

[16] L. Tang, "Weighted norm inequalities for Schrödinger type operators," Forum Mathematicum, vol. 27, pp. 2491-2532, 2015.

[17] Y. S. Jiang, "Some properties of the Riesz potential associated to the Schrödinger operator on the Heisenberg groups," Acta Mathematica Sinica, vol. 53, pp. 785-794, 2010.

[18] Y. S. Jiang, "Endpoint estimates for fractional integral associated to Schrödinger operators on the Heisenberg groups," Acta Mathematica Scientia, vol. 31, no. 3, pp. 993-1000, 2011.

[19] L. Pengtao and P. Lizhong, " $\mathrm{L}^{\mathrm{p}}$ boundedness of commutator operator associated with Schrödinger operators on Heisenberg group," Acta Mathematica Scientia, vol. 32, no. 2, pp. 568-578, 2012.

[20] R. Jiang, X. Jiang, and D. Yang, "Maximal function characterizations of Hardy spaces associated with Schrödinger operators on nilpotent Lie groups," Revista Matemática Complutense, vol. 24, no. 1, pp. 251-275, 2011.

[21] H. Q. Li, "Estimations $\mathrm{L}^{\mathrm{p}}$ des opérateurs de Schrödinger sur les groupes nilpotents," Journal of Functional Analysis, vol. 161, no. 1, pp. 152-218, 1999.

[22] G. Z. Lu, "A Fefferman-Phong type inequality for degenerate vector fields and applications," Panamerican Mathematical Journal, vol. 6, pp. 37-57, 1996.

[23] D. Jerison and A. Sánchez-Calle, "Estimates for the heat kernel for a sum of squares of vector fields," Indiana University Mathematics Journal, vol. 35, no. 4, pp. 835-854, 1986.

[24] J. A. Goldstein, Semigroups of Linear Operators and Applications, Oxford University Press, New York, NY, USA, 1985.

[25] S. Hofmann, G. Lu, D. Mitrea, M. Mitrea, and L. Yan, "Hardy spaces associated to non-negative self-adjoint operators satisfying Davies-Gaffney estimates," Memoirs of the American Mathematical Society, vol. 214, no. 1007, 2011.

[26] T. Coulhon, D. Müller, and J. Zienkiewicz, "About Riesz transforms on the Heisenberg groups," Mathematische Annalen, vol. 305, no. 1, pp. 369-379, 1996.

[27] P. Auscher and B. Ben Ali, "Maximal inequalities and Riesz transform estimates on $\mathrm{L}^{\mathrm{p}}$ spaces for Schrödinger operators with nonnegative potentials," Annales de l'institut Fourier, vol. 57, no. 6, pp. 1975-2013, 2007.

[28] B. Bongioanni, E. Harboure, and O. Salinas, "Commutators of Riesz transforms related to Schrödinger operators," Journal of
Fourier Analysis and Applications, vol. 17, no. 1, pp. 115-134, 2011.

[29] C. B. Morrey, "On the solutions of quasi-linear elliptic partial differential equations," Transactions of the American Mathematical Society, vol. 43, no. 1, pp. 126-166, 1938.

[30] D. R. Adams, Morrey Spaces, Lecture Notes in Applied and Numerical Harmonic Analysis, Birkhäuser/Springer, 2015.

[31] D. R. Adams and J. Xiao, "Morrey spaces in harmonic analysis," Arkiv för Matematik, vol. 50, no. 2, pp. 201-230, 2012.

[32] D. R. Adams and J. Xiao, "Nonlinear potential analysis on Morrey spaces and their capacities," Indiana University Mathematics Journal, vol. 53, pp. 1629-1663, 2004.

[33] F. Chiarenza and M. Frasca, "Morrey spaces and HardyLittlewood maximal function," Rendiconti di Matematica $e$ delle sue Applicazioni, vol. 7, pp. 273-279, 1987.

[34] J. Peetre, "On the theory of $\mathrm{L}_{\mathrm{p}, \lambda}$ spaces," Journal of Functional Analysis, vol. 4, no. 1, pp. 71-87, 1969.

[35] G. Difazio and M. A. Ragusa, "Interior estimates in Morrey spaces for strong solutions to nondivergence form equations with discontinuous coefficients," Journal of Functional Analysis, vol. 112, no. 2, pp. 241-256, 1993.

[36] G. Di Fazio, D. K. Palagachev, and M. A. Ragusa, "Global Morrey regularity of strong solutions to the Dirichlet problem for elliptic equations with discontinuous coefficients," Journal of Functional Analysis, vol. 166, no. 2, pp. 179-196, 1999.

[37] P. Olsen, "Fractional integration, Morrey spaces and a Schrödinger equation," Communications in Partial Differential Equations, vol. 20, no. 11-12, pp. 2005-2055, 1995.

[38] M. E. Taylor, "Analysis on Morrey spaces and applications to Navier-Stokes and other evolution equations," Communications in Partial Differential Equations, vol. 17, no. 9-10, pp. 1407-1456, 1992.

[39] H. Wang, "Morrey spaces related to certain nonnegative potentials and fractional integrals on the Heisenberg groups," Journal of Inequalities and Applications, vol. 2019, no. 1, 2019.

[40] V. S. Guliyev, A. Eroglu, and Y. Y. Mammadov, "Riesz potential in generalized Morrey spaces on the Heisenberg group," Journal of Mathematical Sciences, vol. 189, no. 3, pp. 365-382, 2013.

[41] E. M. Stein and G. Weiss, Introduction to Fourier Analysis on Euclidean Spaces, Princeton University Press, Princeton, NJ, USA, 1971.

[42] Z. Guo, P. Li, and L. Peng, " $\mathrm{L}^{\mathrm{p}}$ boundedness of commutators of Riesz transforms associated to Schrödinger operator," Journal of Mathematical Analysis and Applications, vol. 341, no. 1, pp. 421-432, 2008.

[43] G. B. Folland and E. M. Stein, Hardy Spaces on Homogeneous Groups, Princeton University Press, Princeton, 1982.

[44] J. Xiao and J. He, "Riesz potential on the Heisenberg group," Journal of Inequalities and Applications, vol. 2011, no. 1, Article ID 498638, 2011.

[45] T. Mizuhara, "Boundedness of some classical operators on generalized Morrey spaces," in ICM-90 Satellite Conference Proceedings, S. Igari, Ed., Springer, Tokyo, 1991.

[46] S. Lu, D. Yang, and Z. Zhou, "Sublinear operators with rough kernel on generalized Morrey spaces," Hokkaido Mathematical Journal, vol. 27, pp. 219-232, 1998.

[47] E. Nakai, "Hardy-Littlewood maximal operator, singular integral operators and the Riesz potentials on generalized Morrey spaces," Mathematische Nachrichten, vol. 166, no. 1, pp. 95103, 1994. 